\title{
Limiting forms for surface singularity distributions when the field point is on the surface
}

\author{
T.E. BROCKETT, M.-H. KIM and J.-H. PARK \\ University of Michigan, Department of Naval Architecture and Marine Engineering, Ann Arbor, \\ Michigan 48109, U.S.A.
}

Received 20 April 1988; accepted 31 August 1988

\begin{abstract}
Scalar and vector mathematical identities involving an integral of singularities distributed over a surface and sometimes over a field can be employed to define field values of a quantity of interest. As the volume excluding the singular point from the field tends to zero, the field value is derived. The expressions that result become singular as the point of interest in the field approaches the boundary. Derivation of limiting integral expressions as the field point tends to the surface having a distribution of first and second degree singularities is the main task reported. The limiting expressions for vector values require evaluation as generalized Cauchy Principal-Value Integrals for which some aspect of symmetry in a local region excluding the singularity is required. A contribution from the integral over the local region doubles the value of the identities at a point on the boundary. For a doublet distribution, a singular term arises from the local-region integration that cancels a similar singularity in the integral over the remaining surface. This local contribution for doublets depends explicitly upon the shape of the local region as well as non-orthogonality of the surface coordinate axes. The resulting expressions for surface integrals reproduce known relations for line integrals in two-dimensional fields.
\end{abstract}

\section{Introduction}

Two identities are frequently employed to construct solutions for problems in mathematical physics:

- Green's [10] Third Identity for a scalar (or vector if the gradient of the scalar be taken) field and

- an equation for a vector field that has its antecedents in Stokes' [27] definition of a scalar and vector potential as well as the Maxwell Equations [19] (see also [23], [24] and [5]).

A value of the dependent variable of interest at a point within the field may be constructed in terms of integrals over the surfaces bounding the field as well as over the volume. Often (e.g., for potential flow of an incompressible fluid) the field value is obtained from the integrals over only the bounding surface since equations governing the field give zero values for the weight functions within the volume integrals. For other problems, e.g., the no-slip boundary condition for viscous flow, there is a zero value to the weight function in the surface integral. Considerable literature exists for formulating such solutions for scalar fields when the field point is away from the bounding surfaces (see e.g., [10], [14], [17], [23] and [28]). In general the resulting integrals are well behaved for field points in the volume away from the bounding surface.

To apply boundary conditions or compute certain values of interest on the boundary, limiting forms of the integrals must be derived. The integrand becomes explicitly singular if the field point were set to a point on the boundary. Mangler [18] defines two formalisms that 
may be employed to find the limiting expressions for integrals having an integrand that is singular at a point $\mathbf{r}_{0}$ within or on the domain of integration:

(a) let a field point outside the domain tend to the point $\mathbf{r}_{0}$ within or on the domain, e.g.,

$$
I^{*}\left(\mathbf{r}_{0}\right)=\lim _{\mathbf{r} \rightarrow \mathbf{r}_{0}} I(\mathbf{r})=\lim _{\mathbf{r} \rightarrow \mathbf{r}_{0}}\left[\int_{D} G(\mathbf{r}, s) \mathrm{d} \sigma_{s}\right]=\lim _{\eta \rightarrow 0}\left[I\left(\mathbf{r}_{0}+\eta \mathbf{e}\right)\right]
$$

where $I(\mathbf{r})$ is the integral of $G(\mathbf{r}, s), G(\mathbf{r}, s)$ is singular at $\mathbf{r}=\mathbf{r}_{0}$, a point within or on the domain of integration $D$, and $\mathbf{r}=\mathbf{r}_{0}+\eta \mathbf{e}$ is a point away from the domain of integration (in the present application $\mathbf{r}$ is off a surface or line). We assume that the limit $I^{*}\left(\mathbf{r}_{0}\right)$ as $\mathbf{r} \rightarrow \mathbf{r}_{0}$ is equal to a value known or specified on the boundary, $I\left(\mathbf{r}_{0}\right)$, and do not again call attention to the distinction between $I^{*}\left(\mathbf{r}_{0}\right)$ and $I\left(\mathbf{r}_{0}\right)$.

(b) let the point $\mathbf{r}$ be set to $\mathbf{r}_{0}$ within the domain of integration but exclude a local region about the singular point from the domain, leaving a region of integration $D^{*}$, and take the limit as the excluded region tends to zero (i.e., $D$ becomes an exhaustive extension of $D^{*}$ ):

$$
I\left(\mathbf{r}_{0}\right)=\lim _{D^{*} \rightarrow D}\left[\int_{D^{*}} G\left(\mathbf{r}_{0}, s\right) \mathrm{d} \sigma_{s}\right]
$$

where $D^{*}$ may be only locally different from $D$.

Either of these formalisms for evaluating the integral involve tedious calculations. Examples of approach (b) are the Cauchy integrals in complex-variable analysis, the analysis of Mangler [18], as well as the approach necessary to derive the field values described in the previous paragraph (e.g., [10], [14], [28]). Examples of (a) are in Kellogg [14], MacMillan [17], Mangler [18], and Brockett [5]. Both MacMillan and Kellogg consider a subdivision of a surface (with curvature) into a region cut out by a right circular cylinder aligned with the normal to the surface at the singular point plus the remaining surface. MacMillan [17] is concerned with defining limiting forms that exist as a classical improper integral (e.g., [13]):

(1) determination of the limiting value of the integral as an arbitrary area surrounding the field point tends to zero, and

(2) there be a unique value of the limit as the area of the small region tends to zero.

With a surface subdivision as just defined, MacMillan finds that for a scalar potential involving a distribution of first- or second-degree ${ }^{(1)}$ singularities (e.g., sources, simple, or single-layer distributions and doublet, magnetic shell, or double distributions) over the surface, an improper integral is appropriately derived. MacMillan shows that derivatives of the source and doublet distributions at points on the surface have no "definite meaning" ([17], pp. 155, 174) in the classical improper sense. Kellogg [14] takes the local area to be a near circle, and finds (p. 162) that derivatives of source distributions uniformly approach limits on the boundary, but (p. 168) "does not assert the existence of a limit for the normal derivative" of a doublet distribution.

\footnotetext{
(1) The degree of singularity in the denominator of the integrand as a field point approaches the boundary is defined for two-dimensional fields. The integrand for a surface integral appropriate for a three-dimensional field is increasingly singular by one (that is reduced when integrated over one direction). Specifically source and vortex distributions yield a first-degree singularity and a doublet distribution yields a second-degree singular integral.
} 
The existence of integrals based on only the classical improper criteria is overly restrictive from a practical standpoint, i.e., the mathematically-modeled quantity of interest is expected to exist and generally to be unique on the boundary and the limiting form of the mathematical model should also exist. This viewpoint is to be contrasted with a situation for which an integral is prescribed with a singularity present and the question is one of appropriate evaluation. The approach presented herein is predominately as previously defined in a): to find the limit as a field point tends to a point on the boundary and the integrand becomes singular, but an exhaustive extension of an excluded region is also treated for a special case. In addition to defining a singular integral, a local contribution is obtained. This local contribution is overlooked in some texts on wing theory (e.g., [2], p. 132, and Robinson and Laurmann [25] who, p. 234, unnecessarily, and without discussion of practicality, state a limit as a combined spanwise and chordwise Cauchy Principal Value Integral but do not explicitly develop the limit). The development of limiting forms that proceeds from a subdivision of the bounding surface into a local region that is symmetrical about the singular point plus the remaining surface, then evaluating the limit, first as the field point tends to the surface point and second as the area of the symmetrical region tends to zero, defines a generalized Cauchy Principal Value Integral (CPVI), as opposed to the improper integral often examined in the mathematical literature. Of course, both improper and conventional integrals exist as a CPVI, but not the converse. The preliminary ideas of these more restrictive limiting forms are contained in Hadamard's [11] definition of the finite part of a divergent integral and are refined in the considerations of Muskhilishvili [21] and Mangler [18] who present consideration of the singular forms as Cauchy Principal Value Integrals for two-dimensional fields. Mangler also examines higher-degree singularities.

Glauert [9] uses a cosine transformation for the independent variable and presents an analytic evaluation of the first-degree singular integral useful in numerical analysis of two-dimensional problems. Efficient numerical analysis schemes for a distribution of firstdegree singularities along a curve in a two-dimensional field have been presented based on the Glauert Integral (see, e.g., [22], [29], and [5]) or a polynomial approximation to the numerator of the one-dimensional CPVI with integer exponent $n$, or square root behavior, $n-\frac{1}{2}$, at the ends (e.g., [1] and [15]). Brockett's code [5] evaluates second-degree singularity distributions along a line. Numerical procedures for a planar distribution of first-degree singularities in two- and three-dimensional fields have been proposed with symmetric pairing of the nodes about the singular point or line (see, e.g., respectively, [20] and [26]). Other approaches have been to desingularize the integral (e.g., [16], [6], and [3]). Our concern here is not specifically with this important task of numerical analysis of the equations but with the form of the integral in two-dimensional and three-dimensional space as the field point tends to the bounding surface (hence producing a one-dimensional or two-dimensional surface integral) for which numerical analysis may be required.

The extensive work by investigators such as Mangler [18] and Muskhelishvili [21] has been addressed to two-dimensional fields. The restricted work of Kellogg [14] and MacMillan [17] described previously contains an examination of the limiting form of surface integrals for a three-dimensional field resulting from distributions of source- and doublet-potential singularities over a surface with curvature. More recently, for vector fields limiting forms of integrals have been given by Ebihara ([8], p. 4 \& 6) for source and vortex distributions and Brockett ([4], pp. 26-27) for velocity components from a doublet distribution. The singularity strenths and geometry are assumed to be continuous to as high an order as required 


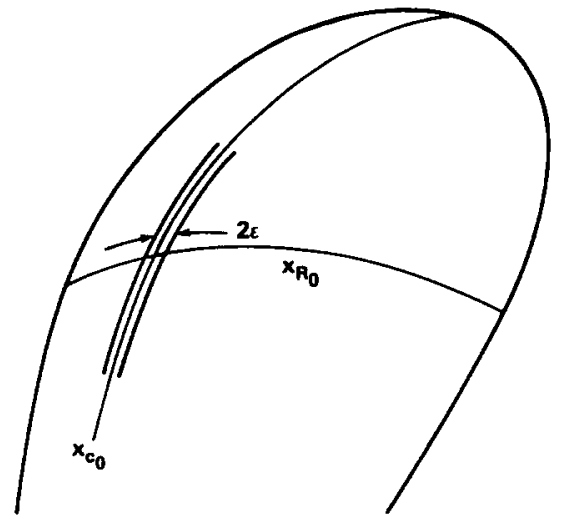

Fig. 1. Schematic of integration regions for limiting forms of singular integrals.

(i.e., be expandable in a Taylor Series about the singular point). Both found it necessary to evaluate the limiting forms as Cauchy Principal Value Integrals. Ebihara [8] gave no clear definition of the meaning of such requirements. Brockett [4] states symmetry of the local region about the singular point is required and defines the effect of the shape of the local region about the singular point for second-degree singularities, but does not consider surface curvature or non-orthogonal coordinate systems in his analysis. In $[17,4]$, mean values are defined for a point on a sheet of discontinuity in the field.

In the present work, one of our major concerns is the derivation of limiting forms of the integrals when the general field point tends to a point $\mathbf{r}_{0}$ on the surface $S$. The surface is divided into a local region about the singular point that we call $\Sigma\left(\mathbf{r}_{0}, \varepsilon\right)$ where $\varepsilon$ characterizes the area of the local region, and the remaining surface $S-\Sigma$. A special concern is to define the form of the limit when $\Sigma$ takes the shape of a "rectangle," $\Sigma\left(\mathbf{r}_{0}, \alpha, \gamma, \delta, \lambda\right)$. Such a shape naturally results when evaluating an improper integral over the remaining surface using variables in the two coordinate directions set up to describe the surface $S-\Sigma$, as indicated schematically in Figs. 1 and 2:

$$
\begin{aligned}
I\left(\mathbf{r}_{0}\right)= & \lim _{\gamma, \alpha \rightarrow 0}\left[\left(\int_{0}^{x_{c 0}-\gamma}+\int_{x_{c 0}+\alpha}^{1}\right) \mathrm{d} x_{c} \int_{0}^{1} G\left(\mathbf{r}_{0}, \mathbf{y}\left(x_{c}, x_{s}\right)\right) \mathrm{d} x_{s}\right. \\
& \left.+\int_{x_{c 0}-\gamma}^{x_{c 0}+\alpha} \mathrm{d} x_{c}\left(\int_{0}^{x_{s 0}-\delta}+\int_{x_{s 0}+\lambda}^{1}\right) G\left(\mathbf{r}_{0}, \mathbf{y}\left(x_{c}, x_{s}\right)\right) \mathrm{d} x_{s}\right]
\end{aligned}
$$

where $x_{c}$ and $x_{s}$ are fraction-of-chord and fraction-of-span coordinates. Of particular interest is to distinguish forms that

and

do not require the second double integral over the narrow open strip in equation (3);

- distinguish between evaluation as a Cauchy Principal Value Integral $(\gamma=\alpha=\varepsilon)$ or as an improper integral.

In addition, the coordinate system defining the surface may be non-orthogonal (e.g., use of fraction-of-chord and span-wise independent variables for a tapered wing or propeller, as 


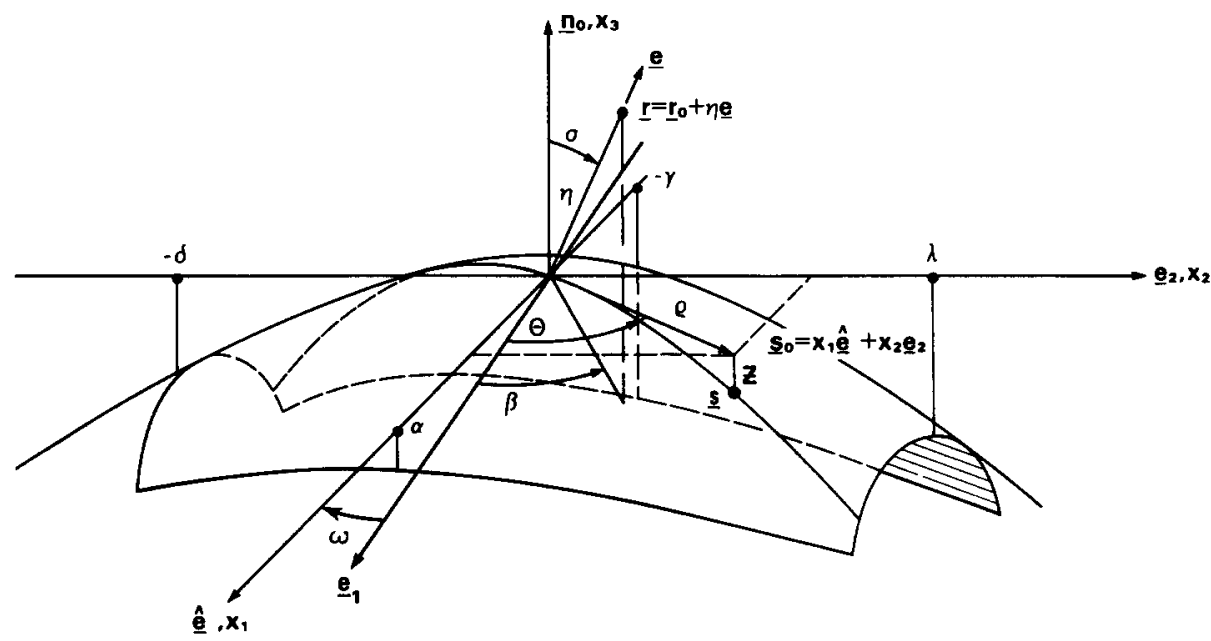

Fig. 2. Coordinate system and surface definition for region surrounding singular point (underlined symbols denote vectors).

indicated in Fig. 1, is a non-orthogonal system) and hence we consider that case. A general schematic of the local region is shown in Fig. 2. A coordinate system is assigned with the origin at the singular point $\mathbf{r}_{0}$ and with two coordinates $\left(x_{1}, x_{2}\right)$ in the plane tangent to the surface at $\mathbf{r}_{0}$ and the third coordinate in the exterior normal direction. Base vectors for the (contravariant) non-orthogonal system are $\left(\hat{\mathbf{e}}, \mathbf{e}_{2}, \mathbf{n}_{0}\right)$, where $\left(\hat{\mathbf{e}}, \mathbf{e}_{2}\right)$ are unit vectors in the tangent plane and $\mathbf{n}_{0}$ is the unit normal at $\mathbf{r}_{0}$ pointing into the field. A reference orthogonal system in the tangent plane is $\left(\mathbf{e}_{1}, \mathbf{e}_{2}\right)$. As $\lambda, \delta$ become large, the strip indicated in Fig. 1 is defined. Since only a short distance in the $x_{2}$ direction is required to have the integral from that point out be of order of the strip width, we sketch $\lambda, \delta$ as of order of the width $(\alpha+\gamma)$ but take the limit as the width vanishes. The surface $S$ bounding $V$ separates a threedimensional space into a field of interest and an "interior" region that is not of concern. In Fig. 2, the surface is shown convex relative to an observer in the field. The determination of the contribution of the integral over this local region to the limiting form of the total integral is the significant investigation described in the main body of the present work.

In general, the results are that:

(1) the limiting forms contain a contribution from the local region $\Sigma$ that doubles the identities (this contribution is not indicated in (3)),

(2) the second integral in equation (3) need not be considered for first-degree singularities and may be eliminated for second-degree singularities by proper choice of $\Sigma$,

(3) the surface integrals for vector quantities require evaluation as a generalized CPVI, and

(4) the limiting integral expressions are not explicitly dependent upon surface curvature.

The limiting form for second-degree singularity (doublet) distributions is found to be influenced by both the shape of the local area about the singular point and by nonorthogonality of the surface coordinates. The limiting form of the integral contains a component from the local region that is singular and cancels a similar singularity in the CPVI over the remaining surface, in agreement with results first defined by Mangler [18] for line 
distributions. These results correct some presentations in the literature and may be of direct use to investigators who require limiting expressions for matched asymptotic expansions as well as those using panel methods and second-degree (doublet) singularities who currently obtain velocity components from differentiation of the potential on the surface rather than compute a consistent panel approximation.

\section{Limiting forms for integrals}

\section{I. Equations for field values}

The starting point for developing equations for field values (point $\mathbf{r} \in V, \mathbf{r} \notin S$ ) of scalar and vector quantities are general identities involving two separate functions derived with the Gauss or Green Divergence Theorem. In these equations, one sets one of the functions equal to an appropriate derivative of the fundamental solution of the Laplace equation, $1 /|\mathbf{r}-\mathbf{s}|$, where the denominator is the distance from the integration point to the field point. For example, Green's Second Identity:

$$
\iiint_{V}\left[\psi \nabla^{2} \varphi-\varphi \nabla^{2} \psi\right] \mathrm{d} V=\oiint_{S}[\varphi \mathbf{n} \cdot \nabla \psi-\psi \mathbf{n} \cdot \nabla \varphi] \mathrm{d} \sigma
$$

with $\psi=1 /|\mathbf{r}-\mathbf{s}|$ and exclusion of a region about $\mathbf{s}=\mathbf{r}$ in the field produces the expression

$$
\lim _{\varepsilon \rightarrow 0}\left[\oiint_{S+S_{B}(\mathbf{r}, \varepsilon)}\left\{\varphi \mathbf{n} \cdot \frac{\mathbf{r}-\mathbf{s}}{|\mathbf{r}-\mathbf{s}|^{3}}-\frac{\mathbf{n} \cdot \nabla \varphi}{|\mathbf{r}-\mathbf{s}|}\right\} \mathrm{d} \sigma_{s}=\lim _{\varepsilon \rightarrow 0} \iiint_{V-B(\mathbf{r}, \varepsilon)} \nabla^{2} \varphi \frac{1}{|\mathbf{r}-\mathbf{s}|} \mathrm{d} V_{s}\right]
$$

where

$S_{B}$ is the surface of a small region $B$ surrounding the fixed singular point $\mathbf{r}$ that is excluded from $V$ to define $V^{*}=V-B$,

$\varphi$ is an arbitrary scalar that is $C^{3}$ continuous at all points within $V$ and on $S$ bounding $V$,

$\mathbf{r}$ is the position vector of a field point within $V$,

$\mathbf{s}$ is the dummy integration variable, a $C^{3}$ continuous point either within $V$ or on the surface $S$, and

$\mathbf{n}$ is the unit normal vector pointing from the surface into the volume.

Similarly a vector identity (See Appendix A of [7]):

$$
\begin{aligned}
& \oiint_{S}[(\mathbf{n} \cdot \mathbf{u}) \mathbf{v}+(\mathbf{n} \times \mathbf{u}) \times \mathbf{v}] \mathrm{d} \sigma \\
& \quad=\iiint_{V}[\mathbf{u} \times(\nabla \times \mathbf{v})+\mathbf{v} \times(\nabla \times \mathbf{u})-\mathbf{v}(\nabla \cdot \mathbf{u})-\mathbf{u}(\nabla \cdot \mathbf{v})] \mathrm{d} V
\end{aligned}
$$




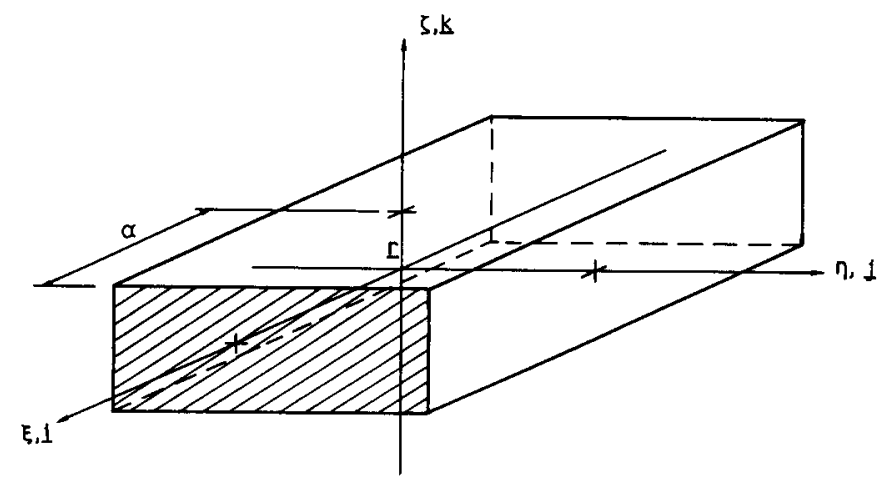

Fig. 3. Parallelepiped excluding singular field point (underlined symbols denote vectors).

leads to an expression for the field value of an arbitrary vector function (with $\mathbf{v}=$ $\left.\nabla_{s}(1 /|\mathbf{r}-\mathbf{s}|)=(\mathbf{r}-\mathbf{s}) /|\mathbf{r}-\mathbf{s}|^{3}\right)$

$$
\begin{aligned}
& \lim _{\varepsilon \rightarrow 0} \oiint_{S+S_{B}}\left[\mathbf{n} \cdot \mathbf{u} \frac{\mathbf{r}-\mathbf{s}}{|\mathbf{r}-\mathbf{s}|^{3}}+(\mathbf{n} \times \mathbf{u}) \times \frac{\mathbf{r}-\mathbf{s}}{|\mathbf{r}-\mathbf{s}|^{3}}\right] \mathrm{d} \sigma_{s} \\
& =-\lim _{\varepsilon \rightarrow 0} \iiint_{V-B(\mathbf{r}, \varepsilon)}\left[\nabla \cdot \mathbf{u} \frac{\mathbf{r}-\mathbf{s}}{|\mathbf{r}-\mathbf{s}|^{3}}+(\nabla \times \mathbf{u}) \times \frac{\mathbf{r}-\mathbf{s}}{|\mathbf{r}-\mathbf{s}|^{3}}\right] \mathrm{d} V_{s} .
\end{aligned}
$$

The vector functions need be only $C^{2}$ continuous in $V$ and on $S$. If $B(\mathbf{r}, \varepsilon)$ were a spherical ball centered at $\mathbf{r}$ with radius $\varepsilon$, the surface integrals over $S_{B}$ can be readily evaluated (for the scalar function see, e.g., [10], [14], [17] or [28]; for the vector function see [7]) and are found to be

$$
\oiint_{S_{B}}(\ldots) \mathrm{d} \sigma=-4 \pi\left\{\begin{array}{l}
\varphi(\mathbf{r}) \text { for the scalar identity } \\
\mathbf{u}(\mathbf{r}) \text { for the vector identity }
\end{array}\right.
$$

The spherical surface describes a symmetrical region about $\mathbf{r}$ and thus defines a Cauchy Principal Value Integral. Here we are concerned about such requirements being necessary or only sufficient, and investigate a different shape to the region about $\mathbf{r}$. This different shape is a parallelepiped, with a right-rectangular cross-section. A sketch of this region is shown in Fig. 3. Consider one face of the parallelepiped at a distance $\xi=\alpha$ from the point $\mathbf{r}$ and let the two Cartesian variables of integration over the surface be $(\eta, \zeta)$. The second term on the left-hand side of the scalar equation (5) can be evaluated on this face:

$$
\begin{aligned}
\iint_{S_{\alpha}}\left[\left.\mathbf{n}\right|_{\alpha} \cdot \nabla \varphi \frac{1}{|\mathbf{r}-\mathbf{s}|}\right] \mathrm{d} \sigma_{s}= & -\int_{\eta_{1}}^{\eta_{2}} \mathrm{~d} \eta \int_{\zeta_{1}}^{\zeta_{2}}\left[\left.\frac{\partial \varphi}{\partial \xi}\right|_{\mathbf{r}}+(\mathbf{s}-\mathbf{r}) \cdot\left\{\left.\nabla \frac{\partial \varphi}{\partial \xi}\right|_{\mathbf{r}}\right\}+\cdots\right] \\
& \times \frac{\mathrm{d} \zeta}{\sqrt{\left(\alpha^{2}+\eta^{2}+\zeta^{2}\right)}} \\
= & -\left[\left.\frac{\partial \varphi}{\partial \xi}\right|_{\mathbf{r}}+\left\{\left.\alpha \frac{\partial^{2} \varphi}{\partial \xi^{2}}\right|_{\mathbf{r}}\right\}\right] \int_{\eta_{1}}^{\eta_{2}} \mathrm{~d} \eta \int_{\zeta_{1}}^{\zeta_{2}} \frac{\mathrm{d} \zeta}{\sqrt{\left(\alpha^{2}+\eta^{2}+\zeta^{2}\right)}} \\
& +\int_{\eta_{1}}^{\eta_{2}} \mathrm{~d} \eta \int_{\zeta_{1}}^{\zeta_{2}} \frac{\mathrm{O}(\eta, \zeta) \mathrm{d} \zeta}{\sqrt{\left(\alpha^{2}+\eta^{2}+\zeta^{2}\right)}} .
\end{aligned}
$$


The first integral is dominant. Let the indefinite integral be

$$
\begin{aligned}
& T_{(1)}(\alpha, \eta, \zeta)=\iint \frac{\mathrm{d} \eta \mathrm{d} \zeta}{\sqrt{\left(\alpha^{2}+\eta^{2}+\zeta^{2}\right)}} \\
& =\alpha\left[\tan ^{-1} \frac{\eta}{\alpha}-\frac{1}{2} \tan ^{-1}\left\{\frac{\eta \zeta}{\alpha} \frac{1}{\sqrt{\left(\alpha^{2}+\zeta^{2}+\eta^{2}\right)}}\right\}\right]+\eta\left[\ln \left\{\zeta+\sqrt{ }\left(\alpha^{2}+\zeta^{2}+\eta^{2}\right)\right\}-1\right] \\
& \quad+\zeta \ln \left[\eta+\sqrt{ }\left(\alpha^{2}+\zeta^{2}+\eta^{2}\right)\right]
\end{aligned}
$$

If $\alpha$ be at a finite distance from the point $\mathbf{r}$ and $(\eta, \zeta)$ tend to zero (i.e., an end to the box with vanishing cross-section), then

$$
\lim _{\eta, \zeta \rightarrow 0}\left[T_{(1)}(\alpha, \eta, \zeta)\right]=0
$$

If $\alpha$ tends to zero (i.e., a side to the shrinking cross-section of the box), then

$$
\begin{aligned}
T_{(1)}^{+}(0, \eta, \zeta) & =\lim _{\alpha \rightarrow 0}\left[T_{(1)}(\alpha, \eta, \zeta)\right] \\
& =\eta\left[\ln \left\{\zeta+\sqrt{ }\left(\zeta^{2}+\eta^{2}\right)\right\}-1\right]+\zeta \ln \left\{\eta+\sqrt{ }\left(\zeta^{2}+\eta^{2}\right)\right\}, \\
T_{(1)}^{-}(0, \eta, \zeta) & =\lim _{\alpha \rightarrow 0}\left[T_{(1)}(-\alpha, \eta, \zeta)\right]=T_{(1)}^{+}(0, \eta, \zeta) .
\end{aligned}
$$

A pair of these side walls of the parallelepiped opposite each other, one at $\alpha_{2}$ and one at $-\alpha_{1}$, have the values as the $\alpha_{\mathrm{i}}$ tend to zero:

$$
\begin{aligned}
& \iint_{S_{x_{2}}}\left[\left.\mathbf{n}\right|_{\alpha_{2}} \cdot \nabla \varphi \frac{1}{|\mathbf{r}-\mathbf{s}|}\right] \mathrm{d} \sigma_{s}+\iint_{S_{\alpha_{1}}}\left[\left.\mathbf{n}\right|_{\alpha_{1}} \cdot \nabla \varphi \frac{1}{|\mathbf{r}-\mathbf{s}|}\right] \mathrm{d} \sigma_{s} \\
& =\left.\frac{\partial \varphi}{\partial \xi}\right|_{\mathbf{r}}\left[T_{(1)}^{+}\left(0, \eta_{2}, \zeta_{2}\right)-T_{(1)}^{+}\left(0, \eta_{2}, \zeta_{1}\right)-\left\{T_{(1)}^{+}\left(0, \eta_{1}, \zeta_{2}\right)-T_{(1)}^{+}\left(0, \eta_{1}, \zeta_{1}\right)\right\}\right] \\
& \quad-\left.\frac{\partial \varphi}{\partial \xi}\right|_{\mathbf{r}}\left[T_{(1)}^{-}\left(0, \eta_{2}, \zeta_{2}\right)-T_{(1)}^{-}\left(0, \eta_{2}, \zeta_{1}\right)-\left\{T_{(1)}^{-}\left(0, \eta_{1}, \zeta_{2}\right)-T_{(1)}^{-}\left(0, \eta_{1}, \zeta_{1}\right)\right\}\right]=0 .
\end{aligned}
$$

Hence, when the $(\eta, \zeta)$ extent of the walls is the same on both the $\alpha_{2}$ and $\alpha_{1}$ faces (i.e., the parallelepiped is right), the integral is zero. Thus there is no contribution from the second term to the value of the integral. 
For the first integral on the left-hand side of the scalar identity, an integral over the face at $\xi=\alpha$, yields

$$
\begin{aligned}
\iint_{S_{\alpha}}\left[\varphi \frac{\left.\mathbf{n}\right|_{\alpha} \cdot(\mathbf{r}-\mathbf{s})}{|\mathbf{r}-\mathbf{s}|^{3}}\right] \mathrm{d} \sigma_{s}= & \varphi\left(\left.\mathbf{r}\right|_{\alpha}\right) \iint_{S_{\alpha}} \frac{-\alpha \mathrm{d} \eta \mathrm{d} \zeta}{\left(\alpha^{2}+\zeta^{2}+\eta^{2}\right)^{3 / 2}}+\text { H.O.T. } \\
= & -\alpha \varphi\left(\left.\mathbf{r}\right|_{\alpha}\right) \iint_{S_{\alpha}} \frac{\mathrm{d} \eta \mathrm{d} \zeta}{\left(\alpha^{2}+\zeta^{2}+\eta^{2}\right)^{3 / 2}}+\text { H.O.T. } \\
= & -\alpha \varphi\left(\left.\mathbf{r}\right|_{\alpha}\right)\left[T_{(2)}\left(\alpha, \eta_{2}, \zeta_{2}\right)-T_{(2)}\left(\alpha, \eta_{1}, \zeta_{2}\right)-T_{(2)}\left(\alpha, \eta_{2}, \zeta_{1}\right)\right. \\
& \left.+T_{(2)}\left(\alpha, \eta_{1}, \zeta_{1}\right)\right]+ \text { H.O.T. }
\end{aligned}
$$

where the indefinite integral is

$$
\begin{aligned}
T_{(2)}(\alpha, \eta, \zeta) & =\iint \frac{\mathrm{d} \eta \mathrm{d} \zeta}{\left(\alpha^{2}+\zeta^{2}+\eta^{2}\right)^{3 / 2}} \\
& =\frac{\zeta}{|\zeta|} \frac{1}{|\alpha|} \tan ^{-1}\left[\frac{\eta|\zeta|}{|\alpha| \sqrt{\left(\alpha^{2}+\zeta^{2}+\eta^{2}\right)}}\right]
\end{aligned}
$$

The four angles in equation (17) are additive positive quantities since $\eta_{1}$ and $\zeta_{1}$ are negative numbers. Also the face at $\xi=-\alpha_{2}$ will produce four angles of the same sign as in equation (17). If $\alpha$ were finite, then

$$
\lim _{\eta, \zeta \rightarrow 0}\left[T_{(2)}(\alpha, \eta, \zeta)\right] \rightarrow 0
$$

Hence, ends (or sides) of the parallelepiped at a finite distance from the singular point do not contribute to the integral. If the side at $\xi=\alpha$ were to approach the singular point (i.e., $\alpha \rightarrow 0$ ) then a careful summation of terms must be assembled. We consider a case such that the sides (all six of them) are all at a distance from the point $\mathbf{r}$ that only the first term of the expansion of $\varphi$ is required and examine the sum of the integrals over the surface $S_{B}$. On each of the six sides of the parallelepiped, there are four arc tangents to be evaluated in equation (17), making a total of twenty four angles to be summed for the integral. If the three angles appropriate for each of the eight corners of the parallelepiped were collected, it is possible to simplify the evaluation. At each vertex there are three angles:

$$
\begin{aligned}
J(\alpha, \eta, \zeta)= & \tan ^{-1}\left[\frac{\eta \zeta}{\alpha} \frac{1}{\sqrt{\left(\alpha^{2}+\eta^{2}+\zeta^{2}\right)}}\right]+\tan ^{-1}\left[\frac{\alpha \zeta}{\eta} \frac{1}{\sqrt{\left(\alpha^{2}+\eta^{2}+\zeta^{2}\right)}}\right] \\
& +\tan ^{-1}\left[\frac{\eta \alpha}{\zeta} \frac{1}{\sqrt{\left(\alpha^{2}+\eta^{2}+\zeta^{2}\right)}}\right]
\end{aligned}
$$

Two of these angles can be added using the relation

$$
\tan ^{-1}(\mathrm{~A})+\tan ^{-1}(\mathrm{~B})=\tan ^{-1}\left(\frac{A+B}{1-A B}\right)=\tan ^{-1}(Q)
$$


where $Q$ is the inverse of the third angle in the expression for $J$ in equation (19). Since the sum of the two acute angles for a right triangle is $\pi / 2$ :

$$
\tan ^{-1}(Q)+\tan ^{-1}(1 / Q)=\pi / 2 .
$$

Since there are eight corners, the total integral has a value

$$
\lim _{\varepsilon \rightarrow 0}\left[\oiint_{S_{B}(\mathbf{r}, \varepsilon)}\left\{\varphi \mathbf{n} \cdot \frac{\mathbf{r}-\mathbf{s}}{|\mathbf{r}-\mathbf{s}|^{3}}\right\}\right] \mathrm{d} \sigma_{s}=-4 \pi \varphi(\mathbf{r})
$$

where $\varepsilon$ need be only a measure of the cross-section dimensions of the parallelepiped, and is required to be sufficiently small that only the first term in the Taylor expansion of $\varphi$ be an accurate representation (on the side walls) of the scalar near $\mathbf{r}$. Similar analysis for $\mathbf{u}(\mathbf{r})$ also produces the same result found when the excluded volume is a sphere as quoted previously.

Thus the equations of interest for our investigation include that from the scalar identity

$$
\varphi(\mathbf{r})=\frac{1}{4 \pi}\left[\oiint_{S}\left\{\varphi \mathbf{n} \cdot \frac{\mathbf{r}-\mathbf{s}}{|\mathbf{r}-\mathbf{s}|^{3}}-\frac{\mathbf{n} \cdot \nabla \varphi}{|\mathbf{r}-\mathbf{s}|}\right\} \mathrm{d} \sigma_{s}-\lim _{\varepsilon \rightarrow 0} \iiint_{V-B(r, s)} \nabla^{2} \varphi \frac{\mathrm{d} V_{s}}{|\mathbf{r}-\mathbf{s}|}\right] .
$$

And for the field value of an arbitrary vector function

$$
\begin{aligned}
\mathbf{u}(\mathbf{r})= & \frac{1}{4 \pi} \oiint_{S}\left[\mathbf{n} \cdot \mathbf{u} \frac{\mathbf{r}-\mathbf{s}}{|\mathbf{r}-\mathbf{s}|^{3}}+(\mathbf{n} \times \mathbf{u}) \times \frac{\mathbf{r}-\mathbf{s}}{|\mathbf{r}-\mathbf{s}|^{3}}\right] \mathrm{d} \sigma_{s} \\
& +\frac{1}{4 \pi} \lim _{\in \rightarrow 0} \iiint_{V-B(r, s)}\left[\nabla \cdot \mathbf{u} \frac{\mathbf{r}-\mathbf{s}}{|\mathbf{r}-\mathbf{s}|^{3}}+(\nabla \times \mathbf{u}) \times \frac{\mathbf{r}-\mathbf{s}}{|\mathbf{r}-\mathbf{s}|^{3}}\right] \mathrm{d} V_{s} .
\end{aligned}
$$

Equation (23) is sometimes called Green's Third Identity ([10] and [14]) and equation (24) is attributed to Poincaré [24] or Philips ([23] p. 199).

For some problems, including the one addressed herein, the gradient of the scalar is also of interest and one is concerned with the following expression:

$$
\begin{aligned}
\nabla \varphi= & \frac{1}{4 \pi}\left[\oiint_{S}\left[\varphi \nabla_{r}\left\{\frac{\mathbf{n} \cdot(\mathbf{r}-\mathbf{s})}{|\mathbf{r}-\mathbf{s}|^{3}}\right\}+\mathbf{n} \cdot \nabla \varphi \frac{\mathbf{r}-\mathbf{s}}{|\mathbf{r}-\mathbf{s}|^{3}}\right] \mathrm{d} \sigma_{2}\right. \\
& \left.+\lim _{\varepsilon \rightarrow 0} \iiint_{V \rightarrow B(\mathbf{r}, z)} \nabla^{2} \varphi \frac{\mathbf{r}-\mathbf{s}}{|\mathbf{r}-\mathbf{s}|^{3}} \mathrm{~d} V_{s}\right]
\end{aligned}
$$

where $B$ may be a sphere or parallelepiped and the gradient term is

$$
\nabla_{r}\left\{\frac{\mathbf{n} \cdot(\mathbf{r}-\mathbf{s})}{|\mathbf{r}-\mathbf{s}|^{3}}\right\}=\frac{\mathbf{n}}{|\mathbf{r}-\mathbf{s}|^{3}}-3 \frac{\mathbf{n} \cdot(\mathbf{r}-\mathbf{s})}{|\mathbf{r}-\mathbf{s}|^{5}}(\mathbf{r}-\mathbf{s}) .
$$


Equations (23), (24) and (25) have interpretations in terms of fluid flow quantities. In equation (23), the first term of the surface integral is equivalent to a distribution of normallydirected doublet singularities of local strength $\varphi(\mathbf{s})$ over the boundary and the second term is equivalent to a distribution of sources (or sinks) of local strength $\mathbf{n} \cdot \nabla \varphi$ over the boundary. In equation (24), the first term of the surface integral is equivalent to the source distribution of equation (23) and the second term is equivalent to a distribution of vortex singularities of local strength $(\mathbf{n} \times \mathbf{u})$ over the boundary.

The equivalence of the doublet and vortex distributions can be shown with the following integral identity ([8], [4] and [12]):

$$
\begin{aligned}
\iint_{S} \varphi \nabla_{r}\left\{\frac{\mathbf{n} \cdot(\mathbf{r}-\mathbf{s})}{|\mathbf{r}-\mathbf{s}|^{3}}\right\} \mathrm{d} \sigma_{s} & =-\iint_{S} \varphi\left(\mathbf{n} \times \nabla_{s}\right) \times \frac{\mathbf{r}-\mathbf{s}}{|\mathbf{r}-\mathbf{s}|^{3}} \mathrm{~d} \sigma_{s} \\
& =\iint_{S}(\mathbf{n} \times \nabla \varphi) \times \frac{\mathbf{r}-\mathbf{s}}{|\mathbf{r}-\mathbf{s}|^{3}} \mathrm{~d} \sigma_{s}+\oint_{c} \varphi \frac{\mathbf{r}-\mathbf{s}}{|\mathbf{r}-\mathbf{s}|^{3}} \times \mathrm{d} \mathbf{l}_{s}
\end{aligned}
$$

where $c$ is the boundary of $S$ and is traversed so that $\mathbf{n}$ points out of the area to the left of an observer. This integral relation shows that the doublet distribution (of strength $\varphi$ ) is equal to the vortex distribution (of strength $\mathbf{n} \times \nabla \varphi$ ) plus a line vortex (of strength $\varphi_{c}$ ) bounding the surface of interest (or that a uniform strength doublet distribution is equal to a line vortex at the boundary of the region of constant strength.) Although this general expression in equation (26) has been recently derived, the equivalent form in Cartesian coordinates for a planar rectangular wing has long been in use (e.g., [2], and [25]).

\subsection{Vector equations as the field position approaches a point on the bounding surface}

Our concern is to define the explicit forms of the surface integrals in equations (24) and (25) as $\mathbf{r}$ tends to $\mathbf{r}_{0}$ a point on the boundary of either a two-dimensional or three-dimensional field. Initially the three-dimensional case will be considered since it is an easy matter to convert the resulting expressions to two-dimensional ones. Specifically, we set out to define the limit as the field point $\mathbf{r}$ approaches a fixed point $\mathbf{r}_{0}$ on the surface bounding the three-dimensional field; i.e., we seek

$$
\lim _{\mathbf{r} \rightarrow \mathbf{r}_{0}^{ \pm}} \nabla \varphi(\mathbf{r})=\lim _{\eta \rightarrow 0} \nabla \varphi\left(\mathbf{r}_{0} \pm \eta \mathbf{e}\right) \equiv \nabla \varphi^{ \pm}\left(\mathbf{r}_{0}\right)
$$

or

$$
\lim _{\mathbf{r} \rightarrow \mathbf{r}_{0}^{ \pm}} \mathbf{u}(\mathbf{r})=\lim _{\eta \rightarrow 0} \mathbf{u}\left(\mathbf{r}_{0} \pm \eta \mathbf{e}\right) \equiv \mathbf{u}^{ \pm}\left(\mathbf{r}_{0}\right)
$$

where $\eta$ is the radius from $\mathbf{r}_{0}, \pm \mathbf{e}$ is an arbitrary vector pointing into the field from $\mathbf{r}_{0}$ (e is always in the field, that is, it never pierces the body), and the \pm sign is used to denote that side of the surface approached at $\mathbf{r}_{0}$. If the surface bounds another volume not of the given field, this distinction of \pm is unnecessary. Distinguishing between \pm sides is an essential consideration for surfaces in the field across which there is a discontinuity. As mentioned in 
the Introduction, the limiting process involved with the surface integrals of scalar function defined in (23) has been treated by Kellogg [14] and MacMillan [17], for a circle surrounding the singular point. A classical improper integral was identified. As shown in the Appendix, a rectangular patch of surface about the surface point also leads to a requirement that the evaluation be improper as opposed to a Cauchy-Principal Value Integral. In addition, the area may be a strip with sides tending to zero rather than having the maximum dimension vanish. In performing the limit process of equation (27), we assume both the function on the surface and the boundary surface description are continuous and differentiable to as high an order as required.

If $\mathbf{r}$ were set directly equal to $\mathbf{r}_{0}$ in the integrands of equations (23), (24), or (25), the integrals would be explicitly singular. To avoid this explicit problem, we consider a limiting process as previously stated:

(1) the surface is divided into two regions, one $\Sigma$ about the singular point, and the other over the remaining area $S-\Sigma$;

(2) in the integral over $S-\Sigma$ (the remaining area) the field point $\mathbf{r}$ can be set to $\mathbf{r}_{0}$ directly;

(3) to perform the integration over the region of the surface surrounding $\mathbf{r}_{0}$, approximations of both the geometry and function appropriate for small depatures from $\mathbf{r}_{0}$ will be made. Once the integral is evaluated, we set $\eta=0$. Next we take the limit $\alpha, \gamma \rightarrow 0$, specify that $\delta, \lambda$ be small so that a Taylor expansion is appropriate, but also take $\alpha /(\delta, \lambda) \rightarrow 0$, $\gamma /(\delta, \lambda) \rightarrow 0$ in the limit as $\alpha, \gamma$ tend to zero. Our notation here is that $(\delta, \lambda)$ means either value in parentheses.

Hence if $\Sigma\left(\mathbf{r}_{0}, \varepsilon\right)$ is the region of $S$ about $\mathbf{r}_{0}$ with characteristic dimension $\varepsilon$, and $\mathbf{G}$ denotes the singular integrand, then the limit process of equation (27) for the integral over the closed surface $S$ is treated as follows:

$$
\lim _{\mathbf{r} \rightarrow \mathbf{r}_{0}} \mathbf{u}(\mathbf{r})=\lim _{\varepsilon \rightarrow 0}\left[\lim _{\eta \rightarrow 0} \iint_{\Sigma\left(\mathbf{r}_{0}, \varepsilon\right)} \mathbf{G}\left(\mathbf{r}_{0}+\eta \mathbf{e}, \mathbf{s}\right) \mathrm{d} \sigma_{s}+\oiint_{S-\Sigma\left(\mathbf{r}_{0}, \varepsilon\right)} \mathbf{G}\left(\mathbf{r}_{0}, \mathbf{s}\right) \mathrm{d} \sigma_{s}\right] .
$$

Figure 1 is a schematic illustration of the area subdivision into $\Sigma$ and $S-\Sigma$ for a reference system that is composed of non-dimensional coordinates for a lifting surface. The singular point is $\left(x_{c 0}, x_{R 0}\right)$ and the selected region $\Sigma$ is a strip of width $2 \varepsilon$ about the $x_{c}$ coordinate line (i.e., $\Sigma$ is bounded by $x_{c}=x_{c 0} \pm \varepsilon$ ). Outside this local region, the integrand is regular and for the most part conventional integration techniques can be employed. However, as shall be shown, the gradient of a doublet distribution has a limit that exists only as indicated in equation (28) and the two separate integrals do not exist independently. As is evident in Fig. 1, the $x_{c 0}$ and $x_{R 0}$ coordinate lines are non-orthogonal and the following analysis will explicitly treat such a case as well as surface curvature. However, geodesic curvature along the $x_{c}$ and $x_{R}$ lines is not considered.

The significant effort involved in equation (28) is the integral over the region $\Sigma$ immediately surrounding $\mathbf{r}_{0}$. Figure 2 is a sketch of such a region cut out of the surface $S$ by a nearly rectangular cylinder having its longitudinal axis aligned with the normal at $\mathbf{r}_{0}$. To approximate the region indicated in Fig. 1, the dimensions $\lambda$ and $\delta$ are arbitrarily large, and the dimensions $\alpha$ and $\gamma$ are both equal to $\varepsilon$.

In Fig. 2, the $\left(x_{1}, x_{2}\right)$ plane is tangent to the surface at $\mathbf{r}_{0}$. The unit vectors $\mathbf{e}_{1}$ and $\mathbf{e}_{2}$ are orthogonal, and $\mathbf{e}$ is in the direction of the variable $x_{1}$. The angle between $\hat{\mathbf{e}}$ and $\mathbf{e}_{1}$ is $\omega$. As 
shown, the surface is convex at $\mathbf{r}_{0}$ when viewed from the field. The exterior normal to $\Sigma$ at $\mathbf{r}_{0}$ is $\mathbf{n}_{0}$ and the $x_{3}$ coordinate is in the $\mathbf{n}_{0}$ direction. In general, the $x_{3}$ coordinate of the patch of surface $\Sigma$ is

$$
x_{3}=-Z\left(x_{1}, x_{2}\right)=-\frac{1}{2}\left\{\frac{x_{1}^{2}}{R_{1}}+2 a_{12} x_{1} x_{2}+\frac{x_{2}^{2}}{R_{2}}\right\}+\cdots
$$

where $R_{1}$ is the radius of curvature of the surface in the $x_{2}=0$ plane, $R_{2}$ is the radius of curvature of the surface in the $x_{1}=0$ plane, and $a_{12}$ depends upon the surface definition. Hence, for the non-orthogonal coordinates $\left(x_{1}, x_{2}, x_{3}\right)$, one has

$$
\mathbf{s}=\mathbf{r}_{0}+\mathbf{s}_{0}-Z \mathbf{n}_{0}=\mathbf{r}_{0}+x_{1} \hat{\mathbf{e}}+x_{2} \mathbf{e}_{2}-Z \mathbf{n}_{0}
$$

and

$$
\mathbf{r}=\mathbf{r}_{0}+\eta \mathbf{e}
$$

where

$$
\begin{aligned}
& \hat{\mathbf{e}}=\cos \omega \mathbf{e}_{1}-\sin \omega \mathbf{e}_{2}, \\
& \begin{aligned}
\mathbf{s}_{0}\left(x_{1}, x_{2}\right) & =x_{1} \hat{\mathbf{e}}+x_{2} \mathbf{e}_{2}=\varrho\left[\cos \theta \mathbf{e}_{1}+\sin \theta \mathbf{e}_{2}\right] \\
& =\varrho\left[\cos \theta / \cos \omega \hat{\mathbf{e}}+(\sin \theta+\cos \theta \tan \omega) \mathbf{e}_{2}\right],
\end{aligned} \\
& \mathbf{e}=\cos \sigma \mathbf{n}_{0}+\sin \sigma\left(\cos \beta \mathbf{e}_{1}+\sin \beta \mathbf{e}_{2}\right) \\
& =\cos \sigma \mathbf{n}_{0}+\sin \sigma\left[\cos \beta / \cos \omega \hat{\mathbf{e}}+(\sin \beta+\cos \beta \tan \omega) \mathbf{e}_{2}\right] .
\end{aligned}
$$

The elemental vector area of integration is

$$
\mathbf{n d} \sigma_{s}=\mathbf{N d} x_{1} \mathrm{~d} x_{2}
$$

where

$$
\begin{aligned}
\mathbf{N} & =\frac{\partial \mathbf{s}}{\partial x_{1}} \times \frac{\partial \mathbf{s}}{\partial x_{2}} \\
& =\cos \omega\left[\mathbf{n}_{0}+\left(\frac{x_{1}}{R_{1}}+a_{12} x_{2}\right) \hat{\mathbf{e}}\right]+\left[a_{12} x_{1}+\frac{x_{2}}{R_{2}}+\sin \omega\left(a_{12} x_{2}+\frac{x_{1}}{R_{1}}\right)\right] \mathbf{n}_{0} \times \hat{\mathbf{e}}
\end{aligned}
$$

An examination of the integrands in equations (23), (24) and (25) shows there are three separate expressions to consider:

$$
\mathbf{I}_{1}=\int_{-\gamma}^{\alpha} \mathrm{d} x_{1} \int_{-\delta}^{\lambda} \mathrm{d} x_{2} \mathbf{N} \cdot \nabla F \frac{\mathbf{r}-\mathbf{s}}{|\mathbf{r}-\mathbf{s}|^{3}}
$$




$$
\begin{aligned}
\mathbf{I}_{2} & =\int_{-\gamma}^{\alpha} \mathrm{d} x_{1} \int_{-\delta}^{\lambda} \mathrm{d} x_{2}(\mathbf{N} \times \nabla F) \times \frac{\mathbf{r}-\mathbf{s}}{|\mathbf{r}-\mathbf{s}|^{3}}, \\
\mathbf{I}_{3} & =\int_{-\gamma}^{\alpha} \mathrm{d} x_{1} \int_{-\delta}^{\lambda} \mathrm{d} x_{2} F \nabla_{r}\left\{\frac{\mathbf{N} \cdot(\mathbf{r}-\mathbf{s})}{|\mathbf{r}-\mathbf{s}|^{3}}\right\} \\
& =\int_{-\gamma}^{\alpha} \mathrm{d} x_{1} \int_{-\delta}^{\lambda} \mathrm{d} x_{2}(\mathbf{N} \times \nabla F) \times \frac{\mathbf{r}-\mathbf{s}}{|\mathbf{r}-\mathbf{s}|^{3}}+\oint_{c} F \frac{\mathbf{r}-\mathbf{s}}{|\mathbf{r}-\mathbf{s}|^{3}} \times \mathrm{d} \mathbf{l}_{s} \\
& =\mathbf{I}_{2}+\mathbf{I}_{4}
\end{aligned}
$$

where $c$ bounds $\Sigma$, and

$$
\mathbf{I}_{4}=\oint_{c} F \frac{\mathbf{r}-\mathbf{s}}{|\mathbf{r}-\mathbf{s}|^{3}} \times \mathrm{dl}_{s} .
$$

In the following we shall consider $\mathbf{I}_{1}, \mathbf{I}_{2}$ and $\mathbf{I}_{4}$ as the three integrals to evaluate although we have also performed the analysis on $\mathbf{I}_{3}$ directly. The advantage of treating $\mathbf{I}_{4}$ is that $\eta$ can be set to zero immediately (i.e., $\mathbf{r}=\mathbf{r}_{0}$ in $\mathbf{I}_{4}$ ).

We first evaluate the $\mathbf{I}_{4}$ integral, since it is simplest and produces the most interesting result. For this integral, the contributions from the $x_{2}=\lambda$ or $x_{2}=-\delta$ portions of the curve bounding the region will vanish in the limit as $\alpha$ and $\gamma$ tend to zero. An expansion of $F$ about $\mathbf{r}_{0}$ is

$$
F(\mathbf{r})=F\left(\mathbf{r}_{0}\right)+\left.\left(\mathbf{s}-\mathbf{r}_{0}\right) \cdot \nabla F\right|_{\mathbf{r}_{0}}+\cdots
$$

Along the side $x_{1}=\alpha$,

$$
d \mathbf{l}_{s}=\left.\frac{\partial \mathbf{s}}{\partial x_{2}}\right|_{x_{1}=\alpha} \mathrm{d} x_{2}=\left[\mathbf{e}_{2}-\left(x_{2} / R_{2}+a_{12} \alpha\right) \mathbf{n}_{0}\right] \mathrm{d} x_{2}+\cdots
$$

and along the side $x_{1}=-\gamma$,

$$
\mathrm{dl}_{s}=-\left.\frac{\partial \mathbf{s}}{\partial x_{2}}\right|_{x_{1}=-\gamma}=-\gamma \mathrm{d} x_{2}=-\left[\mathbf{e}_{2}-\left(x_{2} / R_{2}-a_{12} \gamma\right) \mathbf{n}_{0}\right] \mathrm{d} x_{2}+\cdots
$$

and the integration is from $-\delta$ to $\lambda$ for both terms. Hence

$$
\begin{aligned}
\mathbf{I}_{4}= & F\left(\mathbf{r}_{0}\right) \int_{-\delta}^{\lambda}\left[\mathbf{K}\left(\mathbf{r}_{0}, \alpha, x_{2}\right)-\mathbf{K}\left(\mathbf{r}_{0},-\gamma, x_{2}\right)\right] \mathrm{d} x_{2} \\
& +\nabla F \cdot \hat{\mathbf{e}} \int_{-\delta}^{\lambda}\left[\alpha \mathbf{K}\left(\mathbf{r}_{0}, \alpha, x_{2}\right)+\gamma \mathbf{K}\left(\mathbf{r}_{0},-\gamma, x_{2}\right)\right] \mathrm{d} x_{2} \\
& +\nabla F \cdot \mathbf{e}_{2} \int_{-\delta}^{\lambda} x_{2}\left[\mathbf{K}\left(\mathbf{r}_{0}, \alpha, x_{2}\right)-\mathbf{K}\left(\mathbf{r}_{0},-\gamma, x_{2}\right)\right] \mathrm{d} x_{2} \\
& -\nabla F \cdot \mathbf{n}_{0} \int_{-\delta}^{\lambda}\left[Z\left(\alpha, x_{2}\right) \mathbf{K}\left(\mathbf{r}_{0}, \alpha, x_{2}\right)-Z\left(-\gamma, x_{2}\right) \mathbf{K}\left(\mathbf{r}_{0},-\gamma, x_{2}\right)\right] \mathrm{d} x_{2}+\cdots
\end{aligned}
$$


where

$$
\begin{aligned}
\mathbf{K}\left(\mathbf{r}_{0}, x_{1}, x_{2}\right) & =\frac{\mathbf{r}_{0}-\mathbf{s}\left(x_{1}, x_{2}\right)}{\left|\mathbf{r}_{0}-\mathbf{s}\right|^{3}} \times \frac{\partial \mathbf{s}\left(x_{1}, x_{2}\right)}{\partial x_{2}} \\
& =\frac{\frac{1}{2} \mathbf{e}_{1} / R_{2} x_{2}^{2}-x_{1} / R_{2} \mathbf{n}_{0} \times \hat{\mathbf{e}} x_{2}+\left[\left(-a_{12} \mathbf{n}_{0} \times \hat{\mathbf{e}}-\frac{1}{2} \mathbf{e}_{1} / R_{1}\right) x_{1}-\cos \omega \mathbf{n}_{0}\right] x_{1}}{\left|\mathbf{r}_{0}-\mathbf{s}\right|^{3}} \\
\mathbf{r}_{0}-\mathbf{s}\left(x_{1}, x_{2}\right) & =-x_{1} \hat{\mathbf{e}}-x_{2} \mathbf{e}_{2}+Z \mathbf{n}_{0} .
\end{aligned}
$$

In the denominator, $\left|\mathbf{r}_{0}-\mathbf{s}\right|$ can be approximated by $\left|\mathbf{r}_{0}-\mathbf{s}_{0}\right|$, where $\mathbf{s}_{0}=\mathbf{r}_{0}+x_{1} \hat{\mathbf{e}}+x_{2} \mathbf{e}_{2}$ is the position vector of a point on the tangent plane rather than of a point on the actual surface. The difference between these two surfaces is $Z$, a second-order term, see Kellogg ([14] p. 150) and MacMillan ([17] pp. 169-170). The integrations required in equation (45) are straightforward but tedious. Indefinite integrals listed in the following table are for the non-orthogonal coordinate system and differ slightly from a similar table for orthogonal coordinates although the final result for $\mathbf{I}_{4}$ is identical. The total integral can be constructed from the following elementary integrals:

$$
M_{(1)}^{n}\left(x_{1}, x_{2}\right)=\int \frac{\left(x_{2}\right)^{n}}{\left[A\left(x_{1}\right) x_{2}^{2}+B\left(x_{1}\right) x_{2}+C\left(x_{1}\right)\right]^{3 / 2}} \mathrm{~d} x_{2}
$$

where

$$
A\left(x_{1}\right)=1+\mathrm{O}\left(x_{1}^{2}\right), B\left(x_{1}\right)=-2 \sin \omega x_{1}+\mathrm{O}\left(x_{1}^{3}\right), C\left(x_{1}\right)=x_{1}^{2}+\mathrm{O}\left(x_{1}^{3}\right),
$$

for which

$$
\begin{aligned}
& M_{(1)}^{0}\left(x_{1}, x_{2}\right)=\frac{\operatorname{sign}\left(x_{2}\right)}{x_{1}^{2} \cos ^{2} \omega}-\frac{1}{2 x_{2}\left|x_{2}\right|}+\mathrm{O}\left(x_{1}\right), \\
& M_{(1)}^{1}\left(x_{1}, x_{2}\right)=\frac{\tan \omega}{x_{1} \cos \omega} \operatorname{sign}\left(x_{2}\right)-\frac{1}{\left|x_{2}\right|}+\mathrm{O}\left(x_{1}\right), \\
& M_{(1)}^{2}\left(x_{1}, \lambda\right)-M_{(1)}^{2}\left(x_{1},-\delta\right)=\frac{2\left(2 \sin ^{2} \omega-1\right)}{\cos ^{2} \omega}+\ln \frac{4 \lambda \delta}{x_{1}^{2} \cos ^{2} \omega}+\mathrm{O}\left(x_{1}\right), \\
& M_{(1)}^{3}\left(x_{1}, \lambda\right)-M_{(1)}^{3}\left(x_{1},-\delta\right)=(\lambda-\delta)+\mathrm{O}\left(x_{1} \ln x_{1}\right), \\
& M_{(1)}^{4}\left(x_{1}, \lambda\right)-M_{(1)}^{4}\left(x_{1},-\delta\right)=\frac{1}{2}\left(\lambda^{2}+\delta^{2}\right)+\mathrm{O}\left(x_{1} \ln x_{1}\right) .
\end{aligned}
$$

When the results are combined, the value of $\mathbf{I}_{4}$ as $\alpha \rightarrow 0$ and $\gamma \rightarrow 0$ is obtained from $M_{(1)}^{0}$, $M_{(1)}^{1}$, and $M_{(1)}^{2}$ :

$$
\lim _{\alpha, \gamma \rightarrow 0} \mathbf{I}_{4} \rightarrow\left[-\left(\frac{2}{\alpha}+\frac{2}{\gamma}\right) \frac{\mathbf{n}_{0}}{\cos \omega}-\frac{1}{R_{2}} \ln \frac{\alpha}{\gamma} \mathbf{e}_{1}\right] F\left(\mathbf{r}_{0}\right) .
$$


Hence, it is convenient to take $\gamma=\alpha=\varepsilon$ so that the second term within brackets in (48) vanishes, leaving

$$
\lim _{\varepsilon \rightarrow 0} \mathbf{I}_{4} \rightarrow-\frac{4 \mathbf{n}_{0}}{\varepsilon \cos \omega} F\left(\mathbf{r}_{0}\right)
$$

and no contribution from surface curvature arises but an effect of non-orthogonality is present. If the ends at $x_{2}=-\delta, \lambda$ were at $\delta=\lambda=\varepsilon$, then a different limit would be found. For $\Sigma$ a near square $(\alpha=\lambda=\gamma=\delta=\varepsilon, \omega \neq 0)$ we find

$$
\lim _{\varepsilon \rightarrow 0} \mathbf{I}_{4} \rightarrow-\frac{2 \sqrt{ } 2[\sqrt{ }(1+\sin \omega)+\sqrt{ }(1-\sin \omega)] \mathbf{n}_{0}}{\varepsilon \cos \omega} F\left(\mathbf{r}_{0}\right)
$$

For $\Sigma$ a local area of dimension $\pm \varepsilon$ about both coordinate lines crossing at the point $\mathbf{r}_{0}$ at an angle $\pi / 2 \pm \omega$ :

$$
\lim _{\varepsilon \rightarrow 0} \mathbf{I}_{4} \rightarrow-\frac{8-2 \sqrt{ } 2[\sqrt{ }(1+\sin \omega)+\sqrt{ }(1-\sin \omega)] \mathbf{n}_{0}}{\varepsilon \cos \omega} F\left(\mathbf{r}_{0}\right)
$$

For an orthogonal coordinate system, Brockett ([4], pp. 26-27) gives for the case that $\Sigma$ is a circle:

$$
\lim _{\varepsilon \rightarrow 0} \mathbf{I}_{4} \rightarrow-\frac{2 \pi \mathbf{n}_{0}}{\varepsilon} F\left(\mathbf{r}_{0}\right)
$$

All four of these values (equations 49 and 50) are obviously singular in the limit as $\varepsilon$ tends to zero but cancel similar terms that arise in the integral over $S-\Sigma$. The final result for $\varphi$ is independent of $\Sigma$ but care must be taken to consistently define $\Sigma$. Equations (50) are non-orthogonal generalizations of Brockett's results.

The evaluation of $I_{1}$ and $I_{2}$ is more complicated than $I_{4}$ and involves evaluation of the integral and taking the limit as $\eta$ tends to zero before taking the limit as $\alpha$ and $\gamma$ tend to zero. The denominator can again be approximated as $\mathbf{r}-\mathbf{s}_{0}=\eta \mathbf{e}-x_{1} \hat{\mathbf{e}}-x_{2} \mathbf{e}_{2}$. There result elementary definite integrals with values that can be listed:

$$
M_{(2)}^{m n}=\lim _{\alpha, \gamma \rightarrow 0}\left\{\lim _{\eta \rightarrow 0}\left[\int_{-\gamma}^{\alpha} \mathrm{d} x_{1} \int_{-\delta}^{\lambda} \frac{\left(x_{1}\right)^{m}\left(x_{2}\right)^{n}}{\left[x_{2}^{2}+B\left(x_{1}\right) x_{2}+C\left(x_{1}\right)\right]^{3 / 2}} \mathrm{~d} x_{2}\right]\right\}
$$

where

$$
\begin{aligned}
& B\left(x_{1}\right)=-2 \sin \omega x_{1}-2 \eta \sin \sigma \sin \beta, \\
& C\left(x_{1}\right)=x_{1}^{2}+\eta^{2}-2 \eta x_{1} \sin \sigma \cos (\beta+\omega),
\end{aligned}
$$

for which

$$
M_{(2)}^{00}=\frac{2 \pi}{\eta \cos \omega \cos \sigma}
$$




$$
\begin{aligned}
& M_{(2)}^{01}=2 \pi \tan \sigma \frac{\cos \beta \tan \omega+\sin \beta}{\cos \omega}, \\
& M_{(2)}^{10}=2 \pi \tan \sigma \frac{\cos \beta}{\cos ^{2} \omega}+2 \ln \frac{\alpha}{\gamma}, \\
& M_{(2)}^{02}=M_{(2)}^{20}=M_{(2)}^{11}=0 .
\end{aligned}
$$

Values of $M_{(2)}^{m n}$ with $m, n \geqslant 2$ are zero. The final assembled expression contains $\ln (\alpha / \gamma)$ so that again the equality of $\alpha$ and $\gamma$ is a convenient selection for unambiguous values. Thus

$$
\begin{aligned}
\lim _{\alpha, \gamma \rightarrow 0}\left(\mathbf{I}_{1}\right) & =\cos \omega \mathbf{n}_{0} \cdot \nabla F\left(\mathbf{r}_{0}\right)\left[\eta M_{(2)}^{00} \mathbf{e}-M_{(2)}^{10} \hat{\mathbf{e}}-M_{(2)}^{01} \mathbf{e}_{2}+\mathrm{O}(\eta)\right] \\
& =2 \pi\left[\mathbf{n}_{0} \cdot \nabla F\left(\mathbf{r}_{0}\right)\right] \mathbf{n}_{0} \quad(\text { with } \alpha=\gamma), \\
\lim _{\alpha, \gamma \rightarrow 0}\left(\mathbf{I}_{2}\right) & =\cos \omega\left[\mathbf{n}_{0} \times \nabla F\left(\mathbf{r}_{0}\right)\right] \times\left[\eta M_{(2)}^{00} \mathbf{e}-M_{(2)}^{10} \hat{\mathbf{e}}-M_{(2)}^{01} \mathbf{e}_{2}+\mathrm{O}(\eta)\right] \\
& =2 \pi\left[\nabla F\left(\mathbf{r}_{0}\right)-\mathbf{n}_{0} \cdot \nabla F\left(\mathbf{r}_{0}\right) \mathbf{n}_{0}\right] \quad(\text { with } \alpha=\gamma) .
\end{aligned}
$$

For the sum of $\mathbf{I}_{1}+\mathbf{I}_{2}$, with $\alpha=\gamma=\varepsilon$, we have the local contribution

$$
\lim _{\varepsilon \rightarrow 0}\left(\mathbf{I}_{1}+\mathbf{I}_{2}\right)=\frac{4 \pi}{2} \nabla F\left(\mathbf{r}_{0}\right)
$$

Other shapes for $\Sigma$ (i.e., the square, circle and cross) give identical results. Note an absence of either curvature or explicit local dimensions. It may be that only some aspect of symmetry is appropriate to define an unambiguous value of the integral. Based on the limit for the rectangular region, one has ease of numerical computation by excluding a strip of half width $\varepsilon$ about the coordinate line of interest, as shown in Figure 1.

Collecting the expressions derived, we have the following practical fundamental forms for three-dimensional fields that are both irrotational and solenoidal (i.e., the volume integral need not be considered):

$$
\begin{aligned}
\varphi\left(\mathbf{r}_{0}\right)= & \frac{1}{4 \pi} \lim _{\varepsilon \rightarrow 0}\left[\oiint_{s-\Sigma}\left\{\varphi \mathbf{n} \cdot \frac{\mathbf{r}_{0}-\mathbf{s}}{\left|\mathbf{r}_{0}-\mathbf{s}\right|^{3}}-\frac{\mathbf{n} \cdot \nabla \varphi}{\left|\mathbf{r}_{0}-\mathbf{s}\right|}\right\} \mathrm{d} \sigma_{s}\right]+\frac{1}{2} \varphi\left(\mathbf{r}_{0}\right) \\
\mathbf{u}\left(\mathbf{r}_{0}\right)= & \frac{1}{4 \pi} \lim _{\varepsilon \rightarrow 0}\left[\oiint_{s-\Sigma}\left\{\mathbf{n} \cdot \mathbf{u} \frac{\mathbf{r}_{0}-\mathbf{s}}{\left|\mathbf{r}_{0}-\mathbf{s}\right|^{3}}+(\mathbf{n} \times \mathbf{u}) \times \frac{\mathbf{r}_{0}-\mathbf{s}}{\left|\mathbf{r}_{0}-\mathbf{s}\right|^{3}}\right\} \mathrm{d} \sigma_{s}\right]+\frac{1}{2} \mathbf{u}\left(\mathbf{r}_{0}\right) \\
\nabla \varphi\left(\mathbf{r}_{0}\right)= & \frac{1}{4 \pi} \lim _{\varepsilon \rightarrow 0}\left[\oiint _ { s - \Sigma } \left\{\frac{\mathbf{r}_{0}-\mathbf{s}}{\left|\mathbf{r}_{0}-\mathbf{s}\right|^{3}} \mathbf{n} \cdot \nabla \varphi\right.\right. \\
& \left.\left.+\varphi\left(\frac{\mathbf{n}}{\left|\mathbf{r}_{0}-\mathbf{s}\right|^{3}}-3 \frac{\mathbf{n} \cdot\left(\mathbf{r}_{0}-\mathbf{s}\right)}{\left|\mathbf{r}_{0}-\mathbf{s}\right|^{5}}\left(\mathbf{r}_{0}-\mathbf{s}\right)\right)\right\} \mathrm{d} \sigma_{s}-4 \frac{\mathbf{n}\left(\mathbf{r}_{0}\right) \varphi\left(\mathbf{r}_{0}\right)}{\varepsilon \cos \omega}\right]+\frac{1}{2} \nabla \varphi\left(\mathbf{r}_{0}\right)
\end{aligned}
$$

where $\Sigma$ is a strip of width $2 \varepsilon$ along one coordinate axis passing through $\mathbf{r}_{0}$. 
Two-dimensional expressions can be obtained by extending the spanwise direction (say $x_{2}$, since $\lambda$ and $\delta$ are large) to an infinite but unchanging extent, putting $\omega=0$, and integrating over the spanwise variable. For the gradient of the scalar this procedure results in the expression for two-dimensional fields:

$$
\begin{aligned}
\nabla \varphi\left(\mathbf{r}_{0}\right)= & \frac{1}{2 \pi} \lim _{\varepsilon \rightarrow 0}\left\{\oint_{c-\mathscr{L}}\left[\frac{\mathbf{r}_{0}-\mathbf{s}}{\left|\mathbf{r}_{0}-\mathbf{s}\right|^{2}} \mathbf{n} \cdot \nabla \varphi+\varphi\left(\frac{\mathbf{n}}{\left|\mathbf{r}_{0}-\mathbf{s}\right|^{2}}-2 \frac{\mathbf{n} \cdot\left(\mathbf{r}_{0}-\mathbf{s}\right)}{\left|\mathbf{r}_{0}-\mathbf{s}\right|^{4}}\left(\mathbf{r}_{0}-\mathbf{s}\right)\right)\right] \mathrm{d} l_{s}\right. \\
& \left.-2 \frac{\varphi\left(\mathbf{r}_{0}\right) \mathbf{n}\left(\mathbf{r}_{0}\right)}{\varepsilon}\right\}+\frac{\nabla \varphi\left(\mathbf{r}_{0}\right)}{2}
\end{aligned}
$$

where $\mathscr{L}$ is a arc length $2 \varepsilon$ wide centered at the singular point $\mathbf{r}_{0}$.

Thus for a distribution of only doublets along a straight line from $a$ to $b$, the normal component of the integral reduces to the classical expression [18] for a second-order singularity:

$$
I\left(x_{0}\right)=\lim _{\varepsilon \rightarrow 0}\left[\left(\int_{a}^{x_{0}-\varepsilon}+\int_{x_{0}+\varepsilon}^{b}\right) \frac{F(\xi) \mathrm{d} \xi}{\left|x_{0}-\xi\right|^{2}}-2 \frac{F\left(x_{0}\right)}{\varepsilon}\right] .
$$

A form first defined by Mangler [18] and suggested for use by Ashley and Landahl [2].

\subsection{Gradient of potential as volume excluding surface point vanishes}

A second approach to find a limiting form as $\mathbf{r}$ approaches $\mathbf{r}_{\mathbf{0}}$, as described in the introduction, is to exclude the singular point on the boundary from the field and determine the limit as the excluding volume tends to zero. This approach is particularly simple when the excluded volume is a portion of a sphere centered at the singular point $\mathbf{r}_{0}$ on the boundary. The equation for the gradient of the potential has no singularities when $\mathbf{r}_{0}$ is not within or on $S^{*}\left(S^{*}\right.$ tends to $S$ as a limit to be defined) and the value of the integral over the closed surface $S^{*}$ :

$$
0=\oiint_{S^{*}}\left[\varphi\left\{\frac{\mathbf{n}}{\left|\mathbf{r}_{0}-\mathbf{s}\right|^{3}}-3 \frac{\mathbf{n} \cdot\left(\mathbf{r}_{0}-\mathbf{s}\right)}{\left|\mathbf{r}_{0}-\mathbf{s}\right|^{5}}\left(\mathbf{r}_{0}-\mathbf{s}\right)\right\}+\mathbf{n} \cdot \nabla \varphi \frac{\mathbf{r}_{0}-\mathbf{s}}{\left|\mathbf{r}_{0}-\mathbf{s}\right|^{3}}\right] \mathrm{d} \sigma_{s} .
$$

Let $H$ denote the portion of the surface of the sphere of radius $\varepsilon$ centered at $\mathbf{r}_{0}$ that is within $V$ (see Fig. 4), and $\Sigma$ the region of $S$ interior to $H$. The boundary of $\Sigma$ and $H$ is common. Then

$$
S^{*}=S-\Sigma+H \text {. }
$$

And as the radius $\varepsilon \rightarrow 0, S^{*} \rightarrow S$, and $S-\Sigma$ tends to the area excluding a circle of radius $\varepsilon$ centered at $\mathbf{r}_{0}$. The significant computation is that over the near hemisphere $H$. We use the same spherical polar coordinate system defined in Fig. 2, but now the local area is different. 


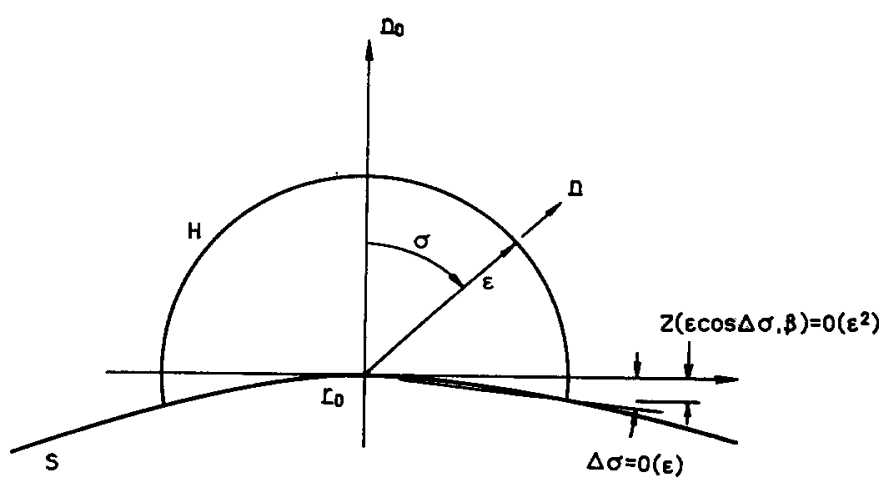

Fig. 4. Cross section of excluded volume centered at a point on the boundary (underlined symbols denote vectors).

The near hemisphere $H$ is defined with spherical coordinates $(\varepsilon, \sigma, \beta)$ where

$$
\begin{aligned}
0 \leqslant \beta \leqslant 2 \pi, 0 \leqslant \sigma \leqslant \pi / 2+\Delta \sigma \\
\Delta \sigma=\tan ^{-1}[Z(\varepsilon \cos \Delta \sigma, \beta) /(\varepsilon \cos \Delta \sigma)] \\
\quad=\frac{1}{2} \varepsilon\left(\cos ^{2} \beta / R_{1}+a_{12} \sin \beta \cos \beta+\sin ^{2} \beta / R_{2}\right)+O\left(\varepsilon^{2}\right)
\end{aligned}
$$

For integration over the surface $H$ we have

$$
\begin{aligned}
& \mathbf{r}_{0}-\mathbf{s}=-\varepsilon \mathbf{n}=-\varepsilon\left(\cos \sigma \mathbf{n}_{0}+\sin \sigma \mathbf{e}_{\beta}\right), \\
& \mathrm{d} \sigma_{s}=\varepsilon^{2} \sin \sigma \mathrm{d} \beta \mathrm{d} \sigma,
\end{aligned}
$$

and

$$
\begin{aligned}
\left.\varphi(\mathbf{r})\right|_{H} & =\varphi\left(\mathbf{r}_{0}\right)+\left.\varepsilon \mathbf{n} \cdot \nabla \varphi\right|_{\mathbf{r}_{0}}+\mathrm{O}\left(\varepsilon^{2}\right) \\
& =\varphi\left(\mathbf{r}_{0}\right)+\varepsilon\left[\left.\mathbf{n}_{0} \cdot \nabla \varphi\right|_{\mathbf{r}_{0}} \cos \sigma+\left.\mathbf{e}_{\beta}(\beta) \cdot \nabla \varphi\right|_{\mathbf{r}_{0}} \sin \sigma\right]+\mathrm{O}\left(\varepsilon^{2}\right)
\end{aligned}
$$

The integrand of the gradient terms in the expansion of $\varphi$ on the surface produces a term that is $-2 \pi \nabla \varphi\left(\mathbf{r}_{0}\right)$, as expected. Since there is nothing new we do not present the computations. The interesting contribution is that involving $\varphi\left(\mathbf{r}_{0}\right)$, resulting from an integration over $H$ :

$$
\begin{aligned}
\mathbf{J} & =\lim _{\varepsilon \rightarrow 0}\left[\varphi\left(\mathbf{r}_{0}\right) \iint_{H}\left\{\frac{\mathbf{n}}{\left|\mathbf{r}_{0}-\mathbf{s}\right|^{3}}-3 \frac{\mathbf{n} \cdot\left(\mathbf{r}_{0}-\mathbf{s}\right)}{\left|\mathbf{r}_{0}-\mathbf{s}\right|^{5}}\left(\mathbf{r}_{0}-\mathbf{s}\right)\right\} \mathrm{d} \sigma_{s}\right] \\
& =\lim _{\varepsilon \rightarrow 0}\left[\varphi\left(\mathbf{r}_{0}\right) \int_{0}^{2 \pi} \mathrm{d} \beta \int_{0}^{1 / 2 \pi+\Delta \sigma}\left\{\frac{\mathbf{n}}{\varepsilon}-3 \frac{\mathbf{n}}{\varepsilon}\right\} \sin \sigma \mathrm{d} \sigma\right] \\
& =-2 \varphi\left(\mathbf{r}_{0}\right) \lim _{\varepsilon \rightarrow 0}\left[\frac{1}{\varepsilon} \int_{0}^{2 \pi} \mathrm{d} \beta \int_{0}^{1 / 2 \pi+\Delta \sigma} \mathbf{n} \sin \sigma \mathrm{d} \sigma\right]
\end{aligned}
$$




$$
\begin{aligned}
& \left.=-2 \varphi\left(\mathbf{r}_{0}\right) \lim _{\varepsilon \rightarrow 0}\left[\frac{1}{\varepsilon} \int_{0}^{2 \pi} \mathrm{d} \beta \int_{0}^{1 / 2 \pi+\Delta \sigma} \cos \sigma \mathbf{n}_{0}+\sin \sigma \mathbf{e}_{\beta}\right) \sin \sigma \mathrm{d} \sigma\right] \\
& =-2 \varphi\left(\mathbf{r}_{0}\right) \lim _{\varepsilon \rightarrow 0}\left[\left.\frac{1}{\varepsilon} \int_{0}^{2 \pi} \mathrm{d} \beta\left\{\frac{1}{2} \sin ^{2} \sigma \mathbf{n}_{0}+\left(\frac{1}{2} \sigma-\frac{1}{4} \sin 2 \sigma\right) \mathbf{e}_{\beta}\right\}\right|_{0} ^{1 / 2 \pi+\Delta \sigma}\right] \\
& =-2 \varphi\left(\mathbf{r}_{0}\right) \lim _{\varepsilon \rightarrow 0}\left[\frac{1}{\varepsilon} \int_{0}^{2 \pi}\left\{\frac{1}{2} \mathbf{n}_{0}+\left(\frac{1}{2} \pi-\Delta \sigma\right) \mathbf{e}_{\beta}+\mathrm{O}\left(\Delta \sigma^{2}\right)\right\} \mathrm{d} \beta\right] \\
& =-2 \varphi\left(\mathbf{r}_{0}\right) \lim _{\varepsilon \rightarrow 0}\left[\frac{\pi \mathbf{n}_{0}}{\varepsilon}-\frac{1}{\varepsilon} \int_{0}^{2 \pi} \Delta \sigma \mathbf{e}_{\beta} \mathrm{d} \beta+\mathrm{O}(\varepsilon)\right] \\
& =-\varphi\left(\mathbf{r}_{0}\right) \lim _{\varepsilon \rightarrow 0}\left[\frac{2 \pi \mathbf{n}_{0}}{\varepsilon}+\mathrm{O}(\varepsilon)\right] .
\end{aligned}
$$

This is the same as the last line of equations (50) and gives an equation for $\nabla \varphi\left(\mathbf{r}_{0}\right)$ :

$$
\begin{aligned}
\frac{1}{2} \nabla \varphi\left(\mathbf{r}_{0}\right)= & \frac{1}{4 \pi} \lim _{\varepsilon \rightarrow 0}\left[\oiint_{s-\Sigma} \varphi\left(\frac{\mathbf{n}}{\left|\mathbf{r}_{0}-\mathbf{s}\right|^{3}}-3 \frac{\mathbf{n} \cdot\left(\mathbf{r}_{0}-\mathbf{s}\right)}{\left|\mathbf{r}_{0}-\mathbf{s}\right|^{5}}\left(\mathbf{r}_{0}-\mathbf{s}\right)\right) \mathrm{d} \sigma_{s}\right. \\
& \left.-2 \pi \frac{\mathbf{n}_{0} \varphi_{0}}{\varepsilon}+\oiint_{s-\Sigma} \mathbf{n} \cdot \nabla \varphi \frac{\mathbf{r}_{0}-\mathbf{s}}{\left|\mathbf{r}_{0}-\mathbf{s}\right|^{3}} \mathrm{~d} \sigma_{s}\right]
\end{aligned}
$$

which is similar to the case for a rectangular region about $\mathbf{r}_{\mathbf{0}}$ quoted in (55) except that a different multiple of the local singularity $1 / \varepsilon$ must be subtracted from the first integral to obtain a finite value, i.e., if (49) and (50) be $K_{\Sigma} F\left(\mathbf{r}_{0}\right) / \varepsilon, K_{\Sigma}$ depends on $\Sigma$. Finally we note that the limiting processes described in (1) and (2) give the same result for this case.

\section{Discussion}

The significant effort presented is the evaluation of the integral over the local region surrounding the singular point in the field or on the body surface. The limiting expression found for scalar and vector identities demonstrates that the scalar (i.e., Green's Third) identity can be computed from the equations derived if only the area of the region excluding the singular point tends to zero. For the vector identities, symmetry as the local region vanishes must be prescribed for unambiguous evaluations.

In the limiting process we assumed that the surface separated a field of interest from a field that was not of concern. Many cases are of this kind but some surfaces are infinitesimally thin (i.e., the shed vortex sheet behind wings) and have a slightly different limit form. For such thin sheets it is easily seen that point value of the variable in the local contribution is replaced by the difference of the potential or vector across the sheet: $\varphi^{+}-\varphi^{-}$, $\nabla\left(\varphi^{+}-\varphi^{-}\right)$, or $\mathbf{u}^{+}-\mathbf{u}^{-}$. Thus the integral produces the mean value on the sheet: $\frac{1}{2}\left(\varphi^{+}+\varphi^{-}\right)$, etc. 
The gradient of the scalar equation has a singularity in the remaining integral that is cancelled with a contribution derived from the evaluation of the integral over the local region about the singular point. This remaining singularity depends upon the shape of the local region. To illustrate this cancellation, we present the following example that is the computation of the self-induced velocity for a rectangular panel over which doublets are distributed. Evaluation over the remaining integral is described and the cancellation relative to the local contribution is demonstrated for two definitions of the local area.

Example: Consider a rectangular patch of surface that is planar and across which there is a distribution of doublets having a constant plus linear variation in strength. Then the normal component of the gradient of $\varphi$ is

$$
w_{n}\left(\mathbf{r}_{0}\right)=\mathbf{n}_{0} \cdot \nabla \varphi=\lim _{\varepsilon \rightarrow 0} \frac{1}{4 \pi}\left[\iint_{s-\Sigma} \frac{\varphi(\mathbf{s}) \mathrm{d} \sigma_{s}}{\left|\mathbf{r}_{0}-\mathbf{s}\right|^{3}}-K_{\Sigma} \frac{\varphi\left(\mathbf{r}_{0}\right)}{\varepsilon}\right]
$$

Construct a coordinate system $\left(x_{1}, x_{2}\right)$ aligned with the edges of the patch and centered at $\mathbf{r}_{0}$, and take the doublet singularity strength as $\varphi(\mathbf{s})=4 \pi\left(1+x_{1}\right)$, then

$$
\begin{aligned}
w_{n}= & \lim _{\varepsilon \rightarrow 0}\left\{\left(\int_{-a}^{-\varepsilon} \mathrm{d} x_{1}+\int_{\varepsilon}^{b} \mathrm{~d} x_{1}\right) \int_{-c}^{d} \frac{\left(1+x_{1}\right) \mathrm{d} x_{2}}{\left[x_{1}^{2}+x_{2}^{2}\right]^{3 / 2}}\right. \\
& \left.+\int_{-\varepsilon}^{\varepsilon} \mathrm{d} x_{1}\left(\int_{-c}^{-\delta} \mathrm{d} x_{2}+\int_{\lambda}^{d} \mathrm{~d} x_{2}\right) \frac{1+x_{1}}{\left[x_{1}^{2}+x_{2}^{2}\right]^{3 / 2}}-K_{\Sigma} \frac{1}{\varepsilon}\right\} \\
= & \lim _{\varepsilon \rightarrow 0}\left\{I_{1}+I_{2}-K_{\Sigma} \frac{1}{\varepsilon}\right\}
\end{aligned}
$$

where

$$
K_{\Sigma}= \begin{cases}4 \sqrt{ } 2 & \text { if } \lambda=\delta=\varepsilon \\ 4 & \text { if } \varepsilon / \lambda, \varepsilon / \delta \rightarrow 0 \text { as } \varepsilon \rightarrow 0\end{cases}
$$

The three indefinite integrals involved with computation of $I_{1}$ and $I_{2}$ are

$$
\begin{aligned}
& \Pi_{1}\left(x_{1}, x_{2}\right)=\int \frac{\mathrm{d} x_{2}}{\left[x_{1}^{2}+x_{2}^{2}\right]^{3 / 2}}=\frac{x_{2}}{x_{1}^{2} \sqrt{\left(x_{1}^{2}+x_{2}^{2}\right)}}, \\
& \Pi_{2}\left(x_{1}, x_{2}\right)=x_{2} \int \frac{\mathrm{d} x_{1}}{x_{1}^{2} \sqrt{ }\left(x_{1}^{2}+x_{2}^{2}\right)}=-\frac{\sqrt{\left(x_{1}^{2}+x_{2}^{2}\right)}}{x_{1} x_{2}}, \\
& \Pi_{3}\left(x_{1}, x_{2}\right)=x_{2} \int \frac{x_{1} \mathrm{~d} x_{1}}{x_{1}^{2} \sqrt{ }\left(x_{1}^{2}+x_{2}^{2}\right)}=-\frac{x_{2}}{\left|x_{2}\right|} \ln \frac{\left|x_{2}\right|+\sqrt{ }\left(x_{1}^{2}+x_{2}^{2}\right)}{\left|x_{1}\right|} .
\end{aligned}
$$


The first definite integral $I_{1}$, over the area $S$ minus the strip $x_{1}= \pm \varepsilon$ becomes

$$
\begin{aligned}
I_{1}(a, b, c, d, \varepsilon)= & -\left(\frac{\sqrt{ }\left(b^{2}+d^{2}\right)}{d b}+\frac{\sqrt{ }\left(a^{2}+d^{2}\right)}{d a}+\frac{\sqrt{ }\left(b^{2}+c^{2}\right)}{c b}+\frac{\sqrt{ }\left(a^{2}+c^{2}\right)}{c a}\right)+\frac{4}{\varepsilon} \\
& +\ln \left[\frac{d+\sqrt{ }\left(a^{2}+d^{2}\right)}{d+\sqrt{ }\left(b^{2}+d^{2}\right)} \frac{b}{a}\right]+\ln \left[\frac{c+\sqrt{ }\left(a^{2}+c^{2}\right)}{c+\sqrt{ }\left(b^{2}+c^{2}\right)} \frac{b}{a}\right]
\end{aligned}
$$

Similarly, for the integral over the strip of width $\pm \varepsilon$ about $x_{1}=0$ :

$$
\begin{aligned}
I_{2}(a, b, c, d, \varepsilon) & =-\frac{4}{\varepsilon}+2 \frac{\sqrt{ }\left(\lambda^{2}+\varepsilon^{2}\right)}{\varepsilon}+2 \frac{\sqrt{ }\left(\delta^{2}+\varepsilon^{2}\right)}{\varepsilon}+\text { H.O.T. } \\
& =-\frac{4}{\varepsilon}+2 \frac{\sqrt{ }\left(1+\varepsilon^{2} / \lambda^{2}\right)}{\varepsilon}+2 \frac{\sqrt{ }\left(1+\varepsilon^{2} / \delta^{2}\right)}{\varepsilon}+\text { H.O.T. }
\end{aligned}
$$

Hence if $\varepsilon / \lambda$ and $\varepsilon / \delta$ tend to zero as $\varepsilon \rightarrow 0$, for which the local region $\Sigma$ excluded in the integral is the strip of width $\pm \varepsilon$ about $x_{1}=0$ :

$$
I_{2}(a, b, c, d, \varepsilon)=0
$$

and if $\varepsilon / \lambda=\varepsilon / \delta=1$, then a square about the singular point $x_{1}=x_{2}=0$ defines the excluded region and

$$
I_{2}(a, b, c, d, \varepsilon)=-\frac{4}{\varepsilon}+\frac{4 \sqrt{ } 2}{\varepsilon} .
$$

The two cases are distinct conceptually but in either case, when the appropriate limit for the local region, $K_{\Sigma}$, is included, the $O(1 / \varepsilon)$ terms cancel and, in the limit as $\varepsilon$ tends to zero, the integral becomes the unambiguous value

$$
\begin{aligned}
w_{n}(a, b, c, d)= & I_{1}(a, b, c, d, \varepsilon)-\frac{4}{\varepsilon} \\
= & -\left(\frac{\sqrt{ }\left(b^{2}+d^{2}\right)}{d b}+\frac{\sqrt{ }\left(a^{2}+d^{2}\right)}{d a}+\frac{\sqrt{ }\left(b^{2}+c^{2}\right)}{c b}+\frac{\sqrt{ }\left(a^{2}+c^{2}\right)}{c a}\right) \\
& +\ln \left[\frac{d+\sqrt{ }\left(a^{2}+d^{2}\right)}{d+\sqrt{ }\left(b^{2}+d^{2}\right)} \frac{b}{a}\right]+\ln \left[\frac{c+\sqrt{ }\left(a^{2}+c^{2}\right)}{c+\sqrt{ }\left(b^{2}+c^{2}\right)} \frac{b}{a}\right]
\end{aligned}
$$

which is a negative number arising from a special integration of a positive integrand.

For the two-dimensional case, $c$ and $d \rightarrow \infty$ and there results an expression easily seen to be equivalent to concentrated vortices of strength $\varphi=4 \pi\left(1+x_{1}\right)$ located at each end of 
the interval, plus a correction (the log term) for a non-centered origin:

$$
\begin{aligned}
w_{n} & =-\left(\frac{1}{b}+\frac{1}{a}+\frac{1}{a}+\frac{1}{b}\right)+2 \ln (b / a) \\
& =-\left(\frac{2}{b}+\frac{2}{a}\right)+2 \ln (b / a) .
\end{aligned}
$$

Thus, the presence of a singularity in the remaining integral term is explicitly derived and subtraction of the appropriate local term, $K_{\Sigma} / \varepsilon$, removes it. In both the evaluation of the local contribution and the integral over the remaining surface integral, a dependence on the shape of the region about the singular point occurs. Also it is evident that the CPVI value is the same as would be obtained if the singularity were ignored for a known indefinite integral. Mangler [18] showed that such results are generally true for one-dimensional line integrals, provided logarithms are computed with the absolute value of the argument. However, one must be careful in interpretation of this statement since a local contribution still doubles the integral value.

\section{Summary}

Expressions for integrals of surface distributions of singularity elements (source, vortex, and dipole) when a field point is a point on the surface bounding the field are derived using two different limiting processes:

- The spatial region about a point on the boundary is excluded from the field of interest, with the point on the surface contained within the excluded local volume. The integral form results as the excluded volume tends to zero.

- The surface is subdivided into a local region about the point on the boundary and the remaining surface. The field point approaches the boundary point.

For the integral over the remaining area, the field point can be set to the boundary point directly, since no singularity explicitly occurs. The integration over the local region is specifically evaluated in the work presented, producing both a doubling of the identities and restrictions on the local shape. The final form of the expression is one for which the local surface region is excluded. Of particular practical interest is the case that the local region is a narrow strip about one of the corrdinate lines (say the spanwise variable) in a reference frame for the surface definition, requiring that only the chordwise integral be addressed with specific analysis for a singularity. For a local area in the shape of such a strip, the remaining integral for the scalar potential identity is improper (only the area need go to zero), but for the vector identities it is a generalized Cauchy Principal-Value Integral (with a strip of width $\pm \varepsilon$ about one of the coordinate lines, as shown in Fig. 1, tending to zero). On sheet discontinuities in the field, the CPVI defines mean values of the dependent variable of the identities.

Limit expressions for the integral identities are given in equation (55). Since there is often concern with integrals of only a single type of singularity element, we list here limiting values 
for them, where $\sigma$ is the scalar source strength, $y$ the vector vortex strength, and $\mu$ the scalar dipole strength:

$$
\begin{aligned}
\mathbf{u}^{\sigma}\left(\mathbf{r}_{0}\right)= & \frac{1}{2} \sigma\left(\mathbf{r}_{0}\right) \mathbf{n}\left(\mathbf{r}_{0}\right)+\frac{1}{4 \pi} \oiint_{S} \sigma \frac{\mathbf{r}_{0}-\mathbf{s}}{\left|\mathbf{r}_{0}-\mathbf{s}\right|^{3}} \mathrm{~d} \sigma_{s}, \\
\mathbf{u}^{\gamma}\left(\mathbf{r}_{0}\right)= & \frac{1}{2} \gamma\left(\mathbf{r}_{0}\right) \times \mathbf{n}\left(\mathbf{r}_{0}\right)+\frac{1}{4 \pi} \oiint_{S} \gamma \times \frac{\mathbf{r}_{0}-\mathbf{s}}{\left|\mathbf{r}_{0}-\mathbf{s}\right|^{3}} \mathrm{~d} \sigma_{s}, \\
\mathbf{w}^{\mu}\left(\mathbf{r}_{0}\right)= & \frac{1}{2}(\mathbf{n} \times \nabla \mu) \times\left.\mathbf{n}\right|_{\mathbf{r}_{0}} \\
& +\frac{1}{4 \pi} \lim _{\varepsilon \rightarrow 0}\left\{\oiint_{S} \mu\left[\frac{\mathbf{n}}{\left|\mathbf{r}_{0}-\mathbf{s}\right|^{3}}-3 \frac{\mathbf{n} \cdot\left(\mathbf{r}_{0}-\mathbf{s}\right)}{\left|\mathbf{r}_{0}-\mathbf{s}\right|^{5}}\left(\mathbf{r}_{0}-\mathbf{s}\right)\right] \mathrm{d} \sigma_{s}-\left.K_{\Sigma} \frac{\mu \mathbf{n}}{\varepsilon}\right|_{\mathbf{r}_{0}}\right\}
\end{aligned}
$$

where $K_{\Sigma}$ is derived from, and has a value dependent on, the shape of the symmetrical local region excluded with the notation $\oiint(-)$ defined to be the integral over $S-\Sigma$ as $\Sigma$ vanishes. For a strip of width $\pm \varepsilon$ about a coordinate line that is one of a pair of non-orthogonal coordinates used to describe the surface, $K_{\Sigma}=4 / \cos \omega$, where $\omega$ is the depature by the coordinate line from a perpendicular to the strip. For vector equations with the seconddegree singularity elements (i.e., doublets), the limiting form specifically depends on the shape of the local area about the point, but the final combined (i.e., local and remaining integral) evaluation is independent of the local shape, as demonstrated in an example computation for a plane rectangular surface. The example also demonstrates the cancellation of singularities within the limiting process that defines the integral.

For the vector quantities involving integrands with a first-degree (i.e., source and vortex elements) singularity at a point on the surface, the limiting form for the integral is the same for a circle, square, cross or rectangular local area. Hence it may be that any local region with symmetry about an axis through the singular point will give the same result.

Two-dimensional forms may be obtained by a spanwise integration.

The equations and perspective presented herein describe limiting expressions that may be addressed for numerical analysis. It is assumed that they exist for a rather broad class of problems that satisfy the continuity conditions specified for equations (5) and (7).

\section{Appendix}

Limiting value of $\varphi(\mathbf{r})$ as $\mathbf{r} \rightarrow \mathbf{r}_{0} \in S$ for the Scalar (Green's Third) Identity

For simplicity, take the surface $\Sigma$ to be a planar rectangular patch described with orthogonal coordinates $\mathbf{s}_{0}-\mathbf{r}_{0}=\left(x_{1}, x_{2}\right)$ as shown in Fig. 5. The integration over a vanishing $\Sigma$ is:

$$
I=\lim _{\alpha, \gamma \rightarrow 0}\left\{\lim _{\mathbf{r} \rightarrow \mathbf{r}_{0}} \frac{1}{4 \pi} \iint_{\Sigma\left(\mathbf{r}_{0}, \alpha, \gamma\right)}\left[\varphi \frac{\mathbf{n} \cdot\left(\mathbf{r}-\mathbf{s}_{0}\right)}{\left|\mathbf{r}-\mathbf{s}_{0}\right|^{3}}+\frac{\mathbf{n} \cdot \nabla \varphi}{\left|\mathbf{r}-\mathbf{s}_{0}\right|}\right] \mathrm{d} \sigma_{s}\right\}
$$

where

$$
\mathbf{r}-\mathbf{s}_{0}=\eta \cos \sigma \mathbf{n}_{0}+\left(\eta \sin \sigma \cos \beta-x_{1}\right) \mathbf{e}_{1}+\left(\eta \sin \sigma \sin \beta-x_{2}\right) \mathbf{e}_{2},
$$




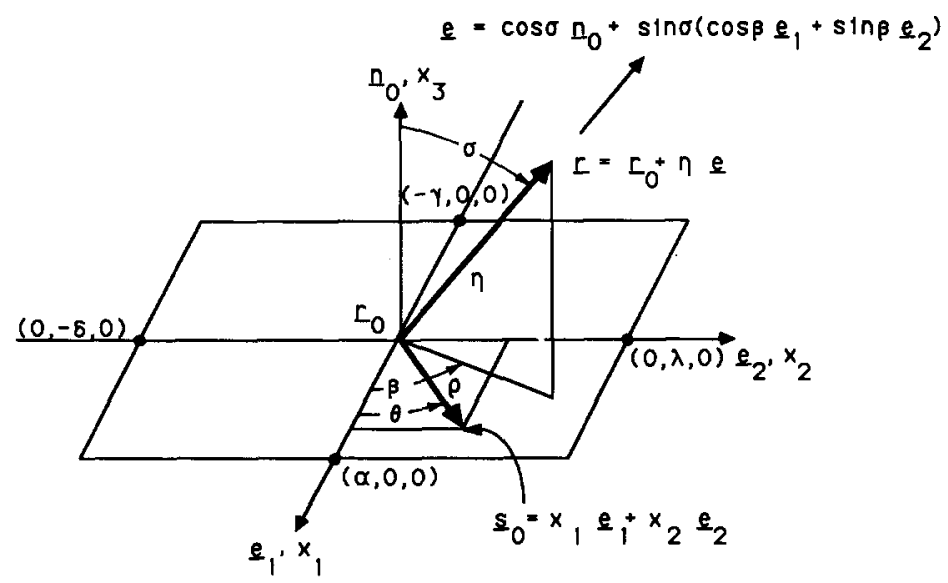

Fig. 5. Planar approximation of surface surrounding a singular point on boundary (underlined symbols denote vectors).

$$
\begin{aligned}
\mathbf{n} \cdot\left(\mathbf{r}-\mathbf{s}_{0}\right) & =\eta \cos \sigma \\
\varphi & =\varphi\left(\mathbf{r}_{0}\right)+\left(\mathbf{s}_{0}-\mathbf{r}_{0}\right) \cdot \nabla \varphi\left(\mathbf{r}_{0}\right)+\cdots \\
& =\varphi\left(\mathbf{r}_{0}\right)+x_{1}\left(\nabla \varphi \cdot \mathbf{e}_{1}\right)_{0}+x_{2}\left(\nabla \varphi \cdot \mathbf{e}_{2}\right)_{0}+\cdots, \\
\mathbf{n} \cdot \nabla \varphi & =\varphi_{n}=\varphi_{n 0}+\left(\mathbf{s}_{0}-\mathbf{r}_{0}\right) \cdot\left(\nabla \varphi_{n}\right)_{0}+\cdots \\
& =\varphi_{n 0}+x_{1}\left(\nabla \varphi_{n} \cdot \mathbf{e}_{1}\right)_{0}+x_{2}\left(\nabla \varphi_{n} \cdot \mathbf{e}_{2}\right)_{0}+\cdots
\end{aligned}
$$

Let

$$
D_{(2)}^{m n}=\lim _{\alpha, \gamma \rightarrow 0}\left[\lim _{\eta \rightarrow 0} \int_{-\gamma}^{\alpha} \mathrm{d} x_{1} \int_{-\delta}^{\lambda} \frac{\left(x_{1}\right)^{m}\left(x_{2}\right)^{n}}{\left[x_{2}^{2}+B x_{2}+C\right]^{3 / 2}} \mathrm{~d} x_{2}\right]
$$

where

$$
B=-2 \eta \sin \sigma \sin \beta, \quad C=x_{1}^{2}+\eta^{2}-2 \eta x_{1} \sin \sigma \cos \beta
$$

Values for $D_{(2)}^{m n}$ are the same as for $M_{(2)}^{m n}$ with $\omega=0$ :

$$
D_{(2)}^{00}=\frac{2 \pi}{\eta \cos \sigma}, \quad D_{(2)}^{01}=2 \pi \tan \sigma \sin \beta, \quad D_{(2)}^{10}=2 \pi \tan \sigma \cos \beta+2 \ln \frac{\alpha}{\gamma}
$$

Thus the first term on the right-hand side of $I$ is

$$
\lim _{\eta \rightarrow 0}\left[\frac{\varphi\left(\mathbf{r}_{0}\right)}{4 \pi}(\eta \cos \sigma) D_{(2)}^{00}\right]=\frac{\varphi\left(\mathbf{r}_{0}\right)}{2}
$$


For the second term on the right-hand side of $I$, let

$$
\begin{aligned}
D_{(1)}^{m n} & =\lim _{\alpha, \gamma \rightarrow 0}\left[\lim _{\eta \rightarrow 0} \iint_{\Sigma} \frac{\left(x_{1}\right)^{m}\left(x_{2}\right)^{n}}{\left|\mathbf{r}-\mathbf{s}_{0}\right|} \mathrm{d} x_{1} \mathrm{~d} x_{2}\right]=\lim _{\alpha, \gamma \rightarrow 0}\left[\lim _{\eta \rightarrow 0} \int_{-\gamma}^{\alpha} \mathrm{d} x_{1} \int_{-\delta}^{\lambda} \frac{\left(x_{1}\right)^{m}\left(x_{2}\right)^{n}}{\left.\sqrt{\left(x_{2}^{2}+B x_{2}+C\right)} \mathrm{d} x_{2}\right]}\right. \\
& =0 \text { for } m=0,1,2, \ldots, n=0,1,2, \ldots
\end{aligned}
$$

Hence

$$
I=\frac{\varphi\left(\mathbf{r}_{0}\right)}{4 \pi} \eta \cos \sigma \frac{2 \pi}{\eta \cos \sigma}=\frac{1}{2} \varphi\left(\mathbf{r}_{0}\right)
$$

Thus there is not a requirement on symmetry of $\Sigma$ to define this value and the result is obtained as a classical improper integral. The local boundaries $\lambda$ and $\delta$ may be arbitrarily large relative to $\alpha$ and $\gamma$. This evaluation of the integral over the local area is included in equation (55).

\section{Acknowledgement}

The material presented herein is a combination of both previous and current work. The new effort was funded by the Office of Naval Research under the Accelerated Research Initiative, "Propulsor-Hull Hydrodynamic Interaction," Contract N00014-86-K-0058.

\section{References}

1. W.E. Alef, Propeller blade sections to be used in a non-homogeneous wake, Hamburg Model Basin, Report 1187 (1959).

2. H. Ashley and M. Landahl, Aerodynamics of Wings and Bodies, Addison-Wesley (1965).

3. M.P. Brandão, Improper integrals in theoretical aerodynamics: The problem revisited, AIAA Journal 25 (1987) 1258-1260.

4. T.E. Brockett, Propeller perturbation problems, U.S. Navy Naval Ship R \& D. Center, Report 3880 (October, 1972).

5. T.E. Brockett, A subroutine for evaluation of one-dimensional singular integrals (SINGINT), Canadian Dept. of National Defence, Defence Research Establishment Atlantic, Tech Memo 79/F (1979).

6. T.E. Brockett, Lifting-surface hydrodynamics for design of rotating blades, Propellers ' 81 Symposium SNAME S-7 (May, 1981).

7. T.E. Brockett, Propeller lifting surface computations, Part 1: Thickness effects, U.S. Navy D.T. Naval Ship R\&D Center, DTNSRDC/SPD-0872/01 (September, 1981).

8. M. Ebihara, A method for the calculation of lifting potential flow problems (1), National Aerospace Laboratory, Japan, TR-240T (July, 1971).

9. H. Glauert, The Elements of Aerofoil and Airscrew Theory, Cambridge (1926).

10. G. Green, An Essay on the Application of Mathematical Analysis to the Theories of Electricity and Magnetism, Nottingham (1828) (Mathematical Papers, Cambridge, 1871, 3-115).

11. J. Hadamard, Lectures on Cauchy's Problem in Linear Partial Differential Equations, New Haven - London, (1923) (also Dover, 1952, 133-141). 
12. J.L. Hess, Calculation of potential flow about arbitrary three-dimensional lifting bodies, McDonnell Douglas Corp., Report J5679-01, (October, 1972).

13. W. Kaplan, Advanced Calculus, Addison-Wesley (1952).

14. O.D. Kellogg, Foundations of Potential Theory, F. Ungar Publishing Co. (1929) (Also Dover, 1953).

15. A. Korneichuk, (1964) As described in Appendix 4 of Bavin, V.F., Zavadovsky, N.Y., Levkovsky, Y.L. and Mishkevitch, V.G., Propellers Modern Methods of Calculation, Izd. Sudostroeniye, Leningrad (in Russian, 1983).

16. L. Landweber, Characteristics of ship boundary layers, 8th Symposium on Naval Hydrodynamics, (1970) 449-475.

17. W.D. MacMillan, The Theory of the Potential, McGraw-Hill (1930) (also Dover, 1958).

18. K.W. Mangler, Improper integrals in theoretical aerodynamics, Royal Aircraft Establishment AERO Report 2424, (June, 1951) (Also ARC C.P. 94, 1952).

19. J.C. Maxell, A Treatise on Electricity and Magnetisms, 2 Vols., Oxford, (1873).

20. V.J. Monacella, On ignoring the singularity in the numerical evaluation of Cauchy Principal Value Integrals, U.S. Navy David Taylor Model Basin, Report 2356, (1967).

21. N.I. Muskhilishvili, Singular Integral Equations, Moscow (1946) (Translation by J.R.M. Radok, Noordhoff, 1953).

22. I. Naiman, Numerical evaluation by harmonic analysis of the $\varepsilon$ function of the Theodorsen arbitrary potential theory, NACA ARR No. L5418, Sept. 1945 (Wartime Report No. L-153, 1945).

23. H.B. Phillips, Vector Analysis, Wiley (1933).

24. H. Poincaré, Théorie du Potentiel Newtonian, Paris (1899).

25. A. Robinson and J.A. Laurmann, Wing Theory, Cambridge (1956).

26. W. Squire, Numerical evaluation of a class of singular double integrals by symmetric pairing, Int. Journal for Numerical Methods in Eng. 10 (1976) 703-708.

27. G.G. Stokes, On the dynamical theory of diffraction, Trans. Cambridge Philosophical Society, 9 (1851) (Math. and Phys. Papers 2, Cambridge, 1880, 243-328).

28. A.H. Tychonov and A.A. Samarski, Partial Differential Equations of Mathematical Physics, Translated by S. Radding, 2 Vols., Holden Day $(1964,1967)$.

29. J. Weber, The calculation of the pressure distribution over the surface of two-dimensional and swept wings with symmetrical aerofoil sections, Aeronautical Research Council R \& M 2918 (1953) (See also R \& M 3026, 1955). 